Supplement of The Cryosphere, 15, 5115-5132, 2021

https://doi.org/10.5194/tc-15-5115-2021-supplement

(C) Author(s) 2021. CC BY 4.0 License.

(c) (i)

Supplement of

\title{
Supraglacial lake bathymetry automatically derived from ICESat-2 constraining lake depth estimates from multi-source satellite imagery
}

Rajashree Tri Datta and Bert Wouters

Correspondence to: Rajashree Tri Datta (tri.datta@gmail.com)

The copyright of individual parts of the supplement might differ from the article licence. 


\section{Supplemental Tables and Figures.}

Lakes Over Sermeq Kujalleq

\begin{tabular}{|c|c|c|c|c|c|c|}
\hline Designation & $\begin{array}{l}\text { Ground } \\
\text { track/spot }\end{array}$ & $\begin{array}{l}\text { Min/Max } \\
\text { Lat }\end{array}$ & $\begin{array}{l}\text { Min/Max } \\
\text { Lon }\end{array}$ & $\begin{array}{l}\text { Max } \\
\text { Lake } \\
\text { Surface } \\
\text { Area } \\
\text { from } \\
\text { imagery } \\
\left(\mathrm{m}^{2}\right)\end{array}$ & $\begin{array}{l}\text { ICESat- } \\
2 \text { track } \\
\text { length } \\
\text { (m) }\end{array}$ & $\begin{array}{l}\text { ICESat- } \\
2 \text { track } \\
\text { Max } \\
\text { depth }\end{array}$ \\
\hline \multicolumn{7}{|c|}{ RGT 727 May $15^{\text {th }}, 2019$} \\
\hline \multicolumn{7}{|c|}{ Landsat: LC08_L1TP_009011_20190514_20190521 } \\
\hline $\begin{array}{l}1 \text { Lake Julian } \\
\text { Coincide with OIB }\end{array}$ & gt11 & $\begin{array}{l}68.707455 \\
68.712048\end{array}$ & $\begin{array}{l}-49.847328 \\
-49.845716\end{array}$ & 160800 & 2754.44 & 3.95 \\
\hline \multicolumn{7}{|c|}{ Sentinel: T22WEB_20190512T151809 T22WEB_20190514T150921 } \\
\hline \multicolumn{7}{|c|}{ RGT 841 May $23^{\text {rd }}$} \\
\hline \multicolumn{7}{|c|}{ Landsat: LC08_L1TP_010011_20190521_20190604_01_T1 } \\
\hline \multicolumn{7}{|c|}{ Sentinel: T22WEB_20190520T152911 T22WEB_20190525T152819 } \\
\hline 1 & gt11_ & $\begin{array}{l}68.616957 \\
68.618372\end{array}$ & $\begin{array}{l}-50.028838 \\
-50.028284\end{array}$ & 357900 & 2398.82 & 1.31 \\
\hline \multicolumn{7}{|c|}{ PlanetScope: 2386345_2270816_2019-05-23_1035_BGRN_SR } \\
\hline \multicolumn{7}{|c|}{ SkySat: 20190522_180624_ssc6d1_0087_analytic } \\
\hline 2 Lake Ayse & gt11 & $\begin{array}{l}68.647653 \\
68.655258\end{array}$ & $\begin{array}{l}-50.041117 \\
-50.038496\end{array}$ & 880300 & 3092.19 & 4.50 \\
\hline \multicolumn{7}{|c|}{$\begin{array}{l}\text { PlanetScope: } \\
\text { 2386345_2270816_2019-05-23_1035_BGRN_SR } \\
\text { 2389698_2270816_2019-05-24_1002_BGRN_SR }\end{array}$} \\
\hline \multicolumn{7}{|c|}{$\begin{array}{l}\text { SkySat: } \\
\text { 20190522_180624_ssc6d1_0082_analytic } \\
\text { 20190522_180624_ssc6d1_0083_analytic }\end{array}$} \\
\hline 3 & gt1r & $\begin{array}{l}68.563169 \\
68.568040\end{array}$ & $\begin{array}{l}-50.009826 \\
-50.008147\end{array}$ & 804873 & 2786.51 & 3.03 \\
\hline $\begin{array}{l}\text { SkySat: } \\
20190522 \\
20190522 \\
\end{array}$ & $\begin{array}{l}\text { ssc6d1__0092 } \\
\text { ssc6d1_009? }\end{array}$ & $\begin{array}{l}\text { analytic } \\
\text { analytic }\end{array}$ & & & & \\
\hline
\end{tabular}




\begin{tabular}{|c|c|c|c|c|c|c|}
\hline 4 & gt1r & $\begin{array}{l}68.646358 \\
68.656471\end{array}$ & $\begin{array}{l}-50.039232 \\
-50.035775\end{array}$ & 880300 & 3372.80 & 4.82 \\
\hline \multicolumn{7}{|c|}{$\begin{array}{l}\text { PlanetScope: } \\
\text { 2386345_2270816_2019-05-23_1035_BGRN_SR } \\
\text { 2389698_2270816_2019-05-24_1002_BGRN_SR }\end{array}$} \\
\hline \multicolumn{7}{|c|}{$\begin{array}{l}\text { SkySat: } \\
\text { 20190522_180624_ssc6d1_0082_analytic } \\
\text { 20190522_180624_ssc6d1_0083_analytic } \\
\text { 20190522_180624_ssc6d1_0084_analytic }\end{array}$} \\
\hline 5 & gt31 & $\begin{array}{l}68.805579 \\
68.808883\end{array}$ & $\begin{array}{l}-49.931074 \\
-49.929887\end{array}$ & 625127 & 2608.30 & 1.81 \\
\hline \multicolumn{7}{|c|}{$\begin{array}{l}\text { PlanetScope: } \\
\text { 20190523_143311_1035_3B_AnalyticMS_SR } \\
\text { 20190524_115042_0f46_3B_AnalyticMS_SR } \\
\text { 20190524_115043_0f46_3B_AnalyticMS_SR }\end{array}$} \\
\hline \multicolumn{7}{|c|}{$\begin{array}{l}\text { SkySat: } \\
\quad \text { 20190522_180624_ssc6d1_0067_analytic }\end{array}$} \\
\hline \multicolumn{7}{|c|}{ RGT 1108 June $9^{\text {th }}, 2019$} \\
\hline \multicolumn{7}{|c|}{ Landsat: LC08_L1TP_008012_20190608_20190619_01_T1 } \\
\hline 1 & gt11 & $\begin{array}{l}68.521802 \\
68.531710\end{array}$ & $\begin{array}{l}-48.870196 \\
-48.866903\end{array}$ & 574200 & 3350.97 & 2.99 \\
\hline 2 & gt1r & $\begin{array}{l}68.521989 \\
68.529861\end{array}$ & $\begin{array}{l}-48.872277 \\
-48.869664\end{array}$ & 574200 & 3123.05 & 1.51 \\
\hline \multicolumn{7}{|c|}{$\begin{array}{l}\text { SkySat: } \\
\text { 20190608_152016_ssc2d3_0080_analytic } \\
\text { 20190608_152016_ssc2d3_0081_analytic }\end{array}$} \\
\hline 3 & gt21 & $\begin{array}{l}68.729626 \\
68.736651\end{array}$ & $\begin{array}{l}-48.881484 \\
-48.879153\end{array}$ & 596931 & 3026.49 & 2.99 \\
\hline \multicolumn{7}{|c|}{$\begin{array}{l}\text { SkySat: } \\
\text { 20190608_152016_ssc2d3_0080_analytic } \\
\text { 20190608_152016_ssc2d3_0081_analytic }\end{array}$} \\
\hline 4 & gt21 & $\begin{array}{l}68.845743 \\
68.855759\end{array}$ & $\begin{array}{l}-48.842804 \\
-48.839477\end{array}$ & 956190 & 3360.29 & 2.18 \\
\hline & $-\operatorname{ssc} 20$ & $\begin{array}{l}\text { analytic } \\
\text { analytic }\end{array}$ & & & & \\
\hline
\end{tabular}




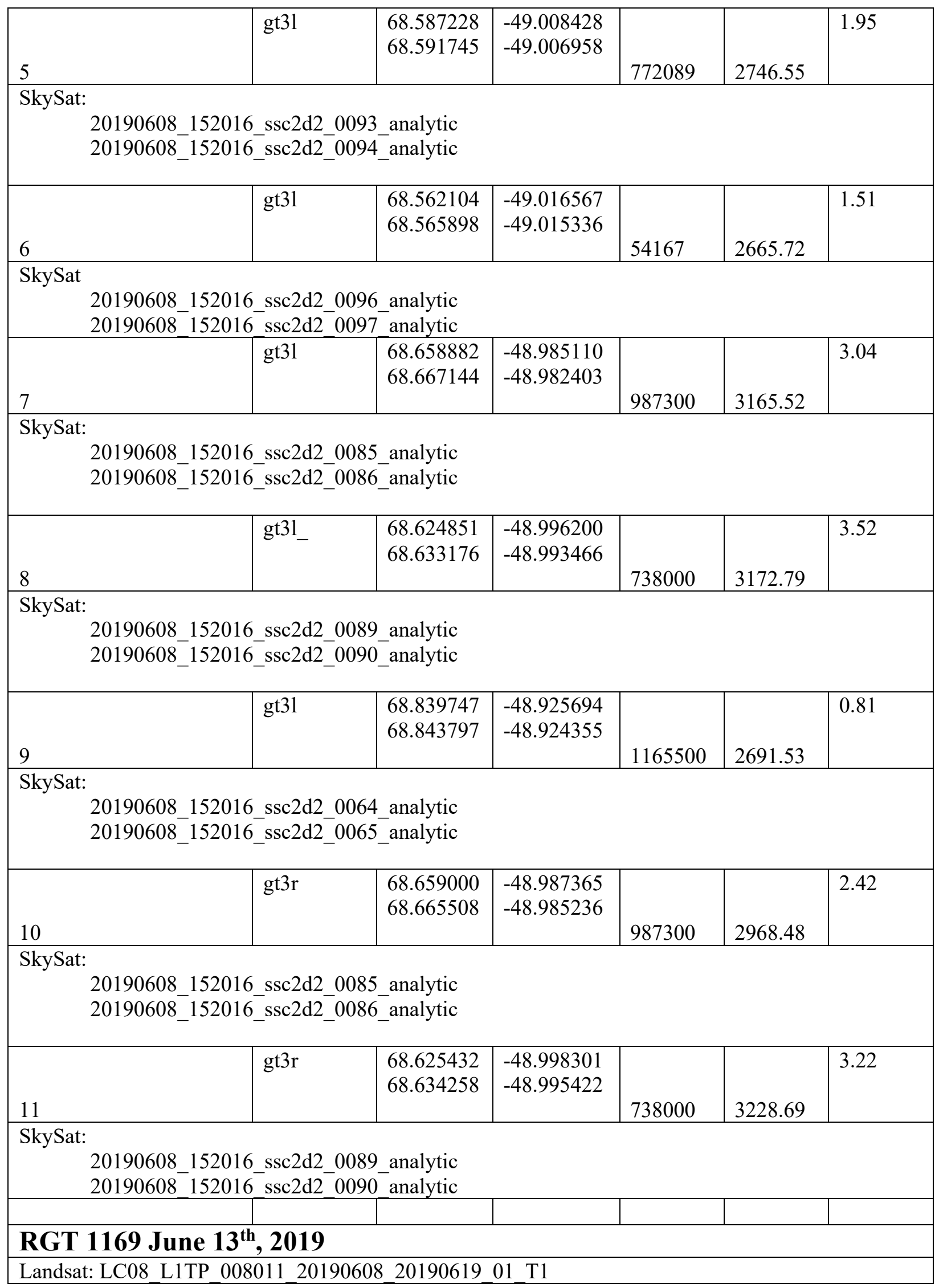




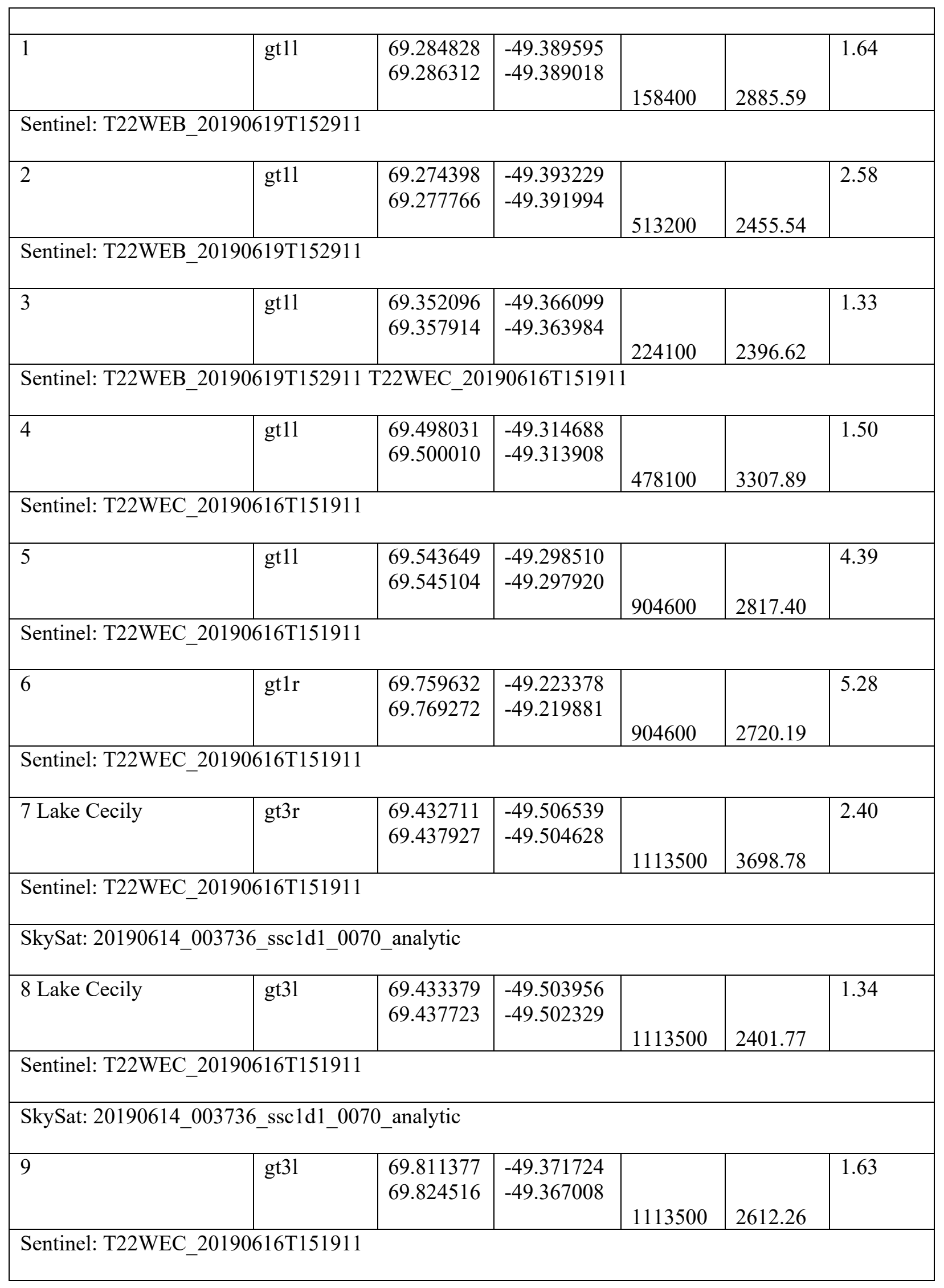




\begin{tabular}{|c|c|c|c|c|c|c|}
\hline \multicolumn{7}{|c|}{ RGT 1222 June $17^{\text {th }}, 2019$} \\
\hline \multicolumn{7}{|c|}{ Landsat: LC08_L1TP_007012_20190617_20190620_01_T1 } \\
\hline 1 & gt11 & $\begin{array}{l}68.456170 \\
68.464562\end{array}$ & $\begin{array}{l}-48.939912 \\
-48.937145\end{array}$ & 2060100 & 3134.91 & 3.62 \\
\hline 2 & gt11 & $\begin{array}{l}68.620541 \\
68.643170\end{array}$ & $\begin{array}{l}-48.999022 \\
-48.991496\end{array}$ & 48600 & 2618.15 & 1.70 \\
\hline 3 & gt11 & $\begin{array}{l}68.704751 \\
68.719064\end{array}$ & $\begin{array}{l}-49.024388 \\
-49.019587\end{array}$ & 1635300 & 4089.09 & 8.93 \\
\hline 4 & gt11 & $\begin{array}{l}68.811525 \\
68.814417\end{array}$ & $\begin{array}{l}-49.056461 \\
-49.055466\end{array}$ & 255600 & 2718.01 & 1.23 \\
\hline 5 & gt1r & $\begin{array}{l}68.620741 \\
68.641831\end{array}$ & $\begin{array}{l}-48.996396 \\
-48.989386\end{array}$ & 1635300 & 4129.21 & 10.62 \\
\hline 6 & gt1r & $\begin{array}{l}68.705881 \\
68.713877\end{array}$ & $\begin{array}{l}-49.020457 \\
-49.017786\end{array}$ & 1692900 & 2679.53 & 1.50 \\
\hline 7 & gt1r & $\begin{array}{l}68.810934 \\
68.814324\end{array}$ & $\begin{array}{l}-49.054218 \\
-49.053076\end{array}$ & 2225700 & 3181.78 & 3.89 \\
\hline 8 & gt21 & $\begin{array}{l}68.427765 \\
68.444251\end{array}$ & $\begin{array}{l}-48.853795 \\
-48.848446\end{array}$ & 1611900 & 4443.29 & 11.43 \\
\hline 9 & gt21 & $\begin{array}{l}68.808374 \\
68.812653\end{array}$ & $\begin{array}{l}-48.975070 \\
-48.973635\end{array}$ & 998574 & 2541.70 & 2.14 \\
\hline 10 & gt2r & $\begin{array}{l}68.428276 \\
68.445114\end{array}$ & $\begin{array}{l}-48.851875 \\
-48.846413\end{array}$ & 998574 & 2802.32 & 1.70 \\
\hline 11 & gt2r & $\begin{array}{l}68.523066 \\
68.526979\end{array}$ & $\begin{array}{l}-48.878531 \\
-48.877251\end{array}$ & 1025100 & 3193.46 & 2.86 \\
\hline \multicolumn{7}{|c|}{$\begin{array}{l}\text { SkySat: } \\
\text { 20190616_144210_ssc4d2_0122_analytic } \\
\text { 20190616_144210_ssc4d2_0123_analytic } \\
\text { 20190616_144210_ssc4d2_0124_analytic } \\
\text { 20190617_152915_ssc1d2_0122_analytic } \\
\text { 20190617_152915_ssc1d2_0123_analytic }\end{array}$} \\
\hline 12 & gt31 & $\begin{array}{l}68.443954 \\
68.463598\end{array}$ & $\begin{array}{l}-48.780603 \\
-48.774288\end{array}$ & 1611900 & 4210.25 & 11.55 \\
\hline 13 & gt31 & $\begin{array}{l}68.621338 \\
68.624034\end{array}$ & $\begin{array}{l}-48.832513 \\
-48.831615\end{array}$ & 1025100 & 3860.27 & 1.59 \\
\hline
\end{tabular}




\begin{tabular}{|c|c|c|c|c|c|c|}
\hline 14 Lake Zadie & gt31 & $\begin{array}{l}68.731742 \\
68.736769\end{array}$ & $\begin{array}{l}-48.869345 \\
-48.867678\end{array}$ & 4101300 & 4774.88 & 11.49 \\
\hline \multicolumn{7}{|c|}{$\begin{array}{l}\text { SkySat: } \\
\text { 20190616_144210_ssc4d3_0092_analytic } \\
\text { 20190616_144210_ssc4d3_0093_analytic }\end{array}$} \\
\hline 15 & gt31 & $\begin{array}{l}68.837005 \\
68.845532\end{array}$ & $\begin{array}{l}-48.905172 \\
-48.902345\end{array}$ & 2060100 & 3842.27 & 3.08 \\
\hline 16 & gt3r & $\begin{array}{l}68.445097 \\
68.462664\end{array}$ & $\begin{array}{l}-48.778073 \\
-48.772433\end{array}$ & 998574 & 2562.81 & 0.35 \\
\hline 17 & gt3r & $\begin{array}{l}68.835768 \\
68.850254\end{array}$ & $\begin{array}{l}-48.904473 \\
-48.899674\end{array}$ & 4101300 & 4602.40 & 10.64 \\
\hline 18 & gt31 & $\begin{array}{l}69.614426 \\
69.630295\end{array}$ & $\begin{array}{l}-49.172278 \\
-49.166720\end{array}$ & 5656500 & 4005.92 & 6.13 \\
\hline \multicolumn{7}{|c|}{ Landsat: LC08_L1TP_008011_20190608_20190619_01_T1 } \\
\hline \multicolumn{7}{|c|}{ Sentinel: T22WEC_20190616T151911 } \\
\hline 19 & gt31 & $\begin{array}{l}69.645719 \\
69.651690\end{array}$ & $\begin{array}{l}-49.179791 \\
-49.177687\end{array}$ & 2982600 & 2899.97 & 1.70 \\
\hline \multicolumn{7}{|c|}{ Landsat: LC08_L1TP_008011_20190608_20190619_01_T1 } \\
\hline Sentinel: T22 & 167 & & & & & \\
\hline
\end{tabular}

Table S1: Lakes used in this study noting Lake number, ICEsat-2 details (RGT/ground track/spot), maximum latitude and longitude boundaries for the lake, maximum surface area of the lake detected from imagery and the length of the ICESat-2 pass over the lake. Concurrent imagery sources with unique identifiers are shown below each lake (with imagery associated with the full RGT noted blow each RGT/date designation).. Lake numbers correspond to profiles shown in Supplemental Figure 3. 


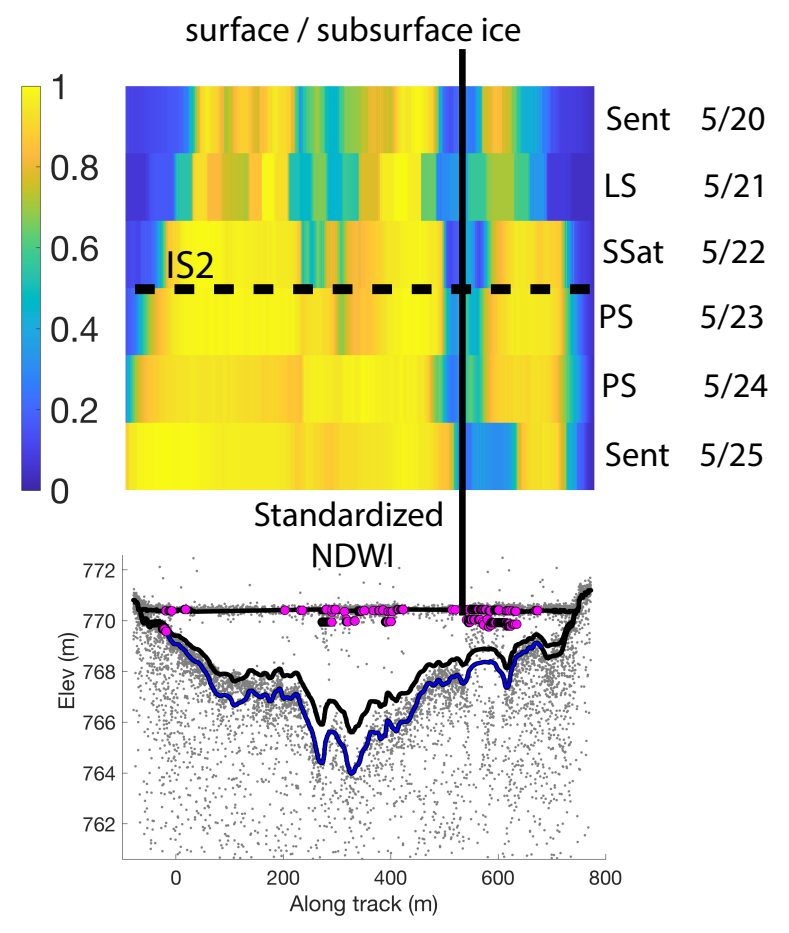

(a) Lake Amitav NDWI

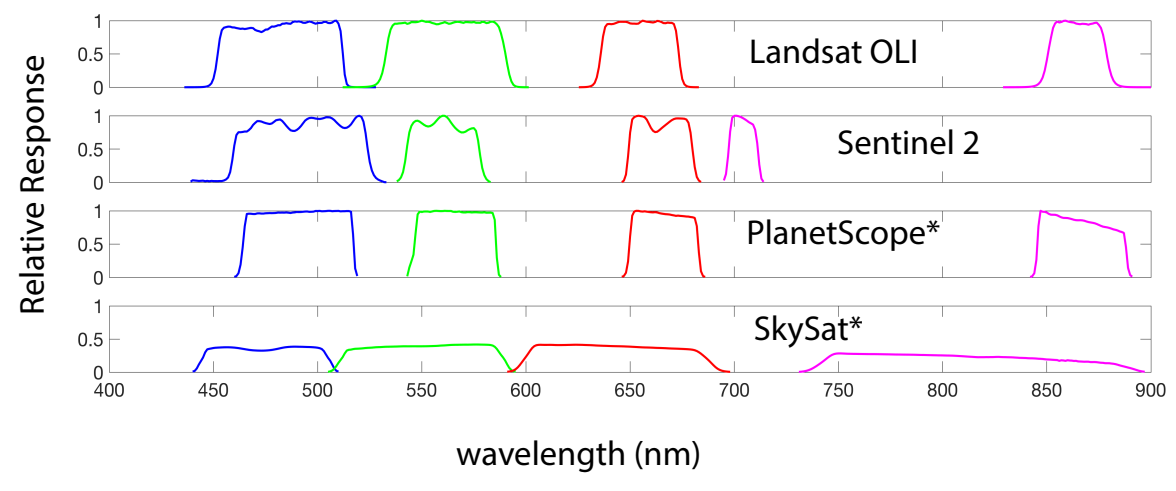

(b) Spectral Response

Figure S1: (a) Watta calculated profile of depth, depth corrected for refraction and subsurface ice over Lake Amitav (bottom) with NDWI values co-registered to the ICESat-2 track (top) (b) Spectral Response curve for imagery sources used in main text. Note that PlanetScope and SkySat data are shown for the satellite used in imagery collected over Lake Amitav and are not representative of the entire constellation. Landsat OLI source (Barsi et al., 2014)., Sentinel-2 source (https:/learth.esa.int/web/sentinel/userguides/sentinel-2-msi), Planet Labs SkySat and PlanetScope (see: support.planet.com) 

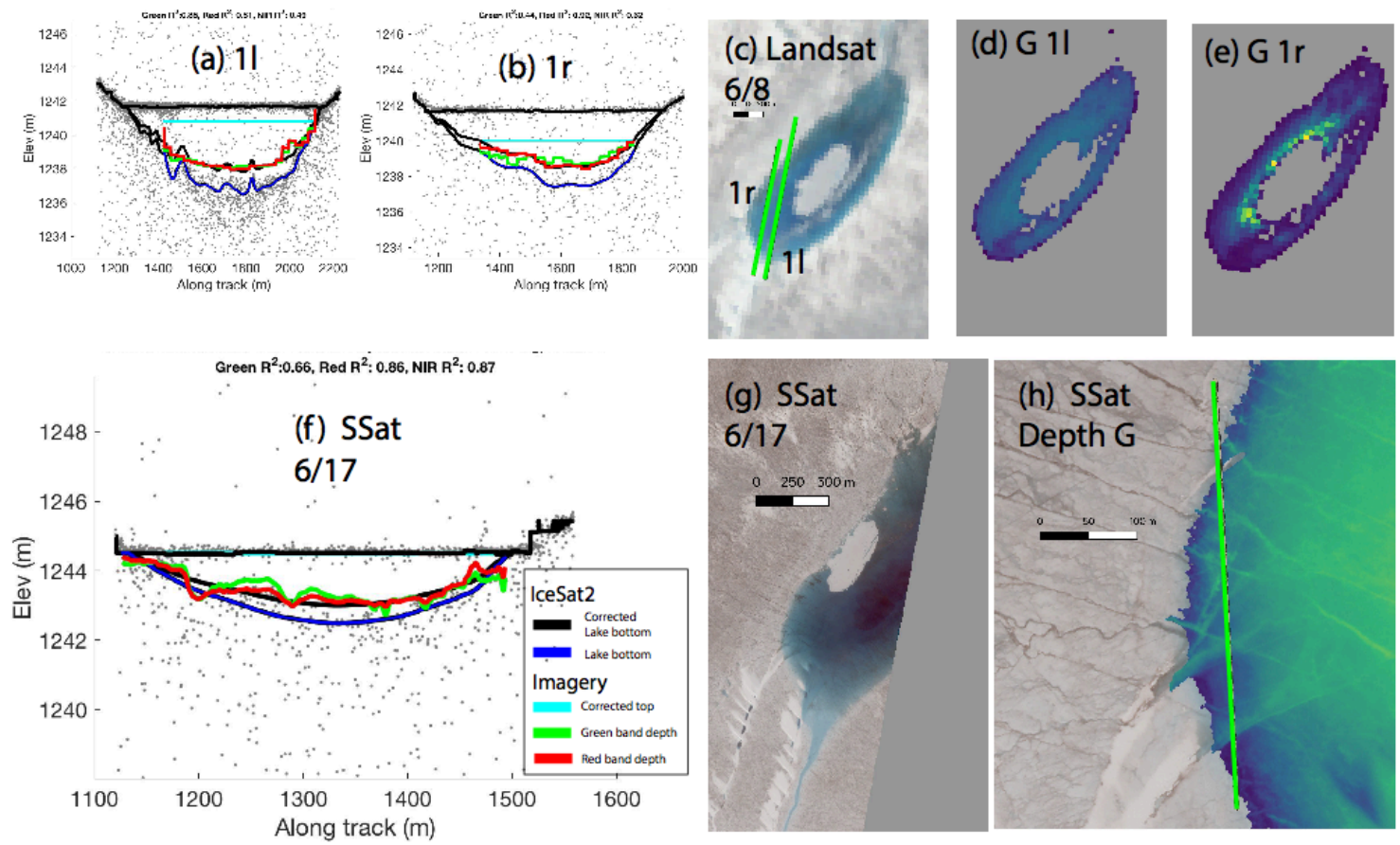

Figure S2: Watta-calculated depth, corrected depth and lake surface profiles for (top) RGT 1108 Lake 1 on June $9^{\text {th }}, 2019$, with gt1l (a,d) and gt1r (b,e). Landsat imagery collected on June $8^{\text {th }}$, 2019. (bottom) The same lake, also RGT 1222 Lake 6 on June $17^{\text {th }}, 2019$ (f-h), with SkySat imagery collected on June $17^{\text {th }}, 2019$. 
Figure: S3. Lake Depth Profiles (corresponding to Table S1)

RGT 727

May $15^{\text {th }}, 2019$

Lake 1:

Sentinel-2: May 12 ${ }^{\text {th }}, 2019$

Sentinel-2: May $14^{\text {th }}, 2019$
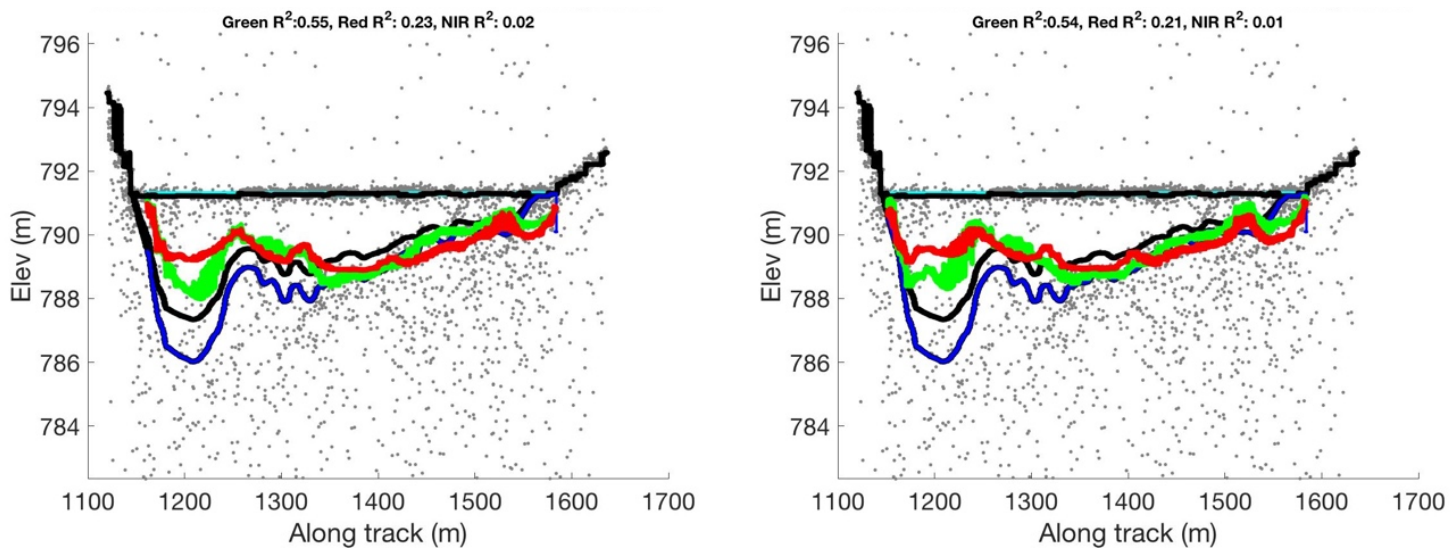

IceSat2

Corrected
Lake bottom
Lake bottom
Imagery
Corrected top
Green band depth
Red band depth




\section{Lake 1}
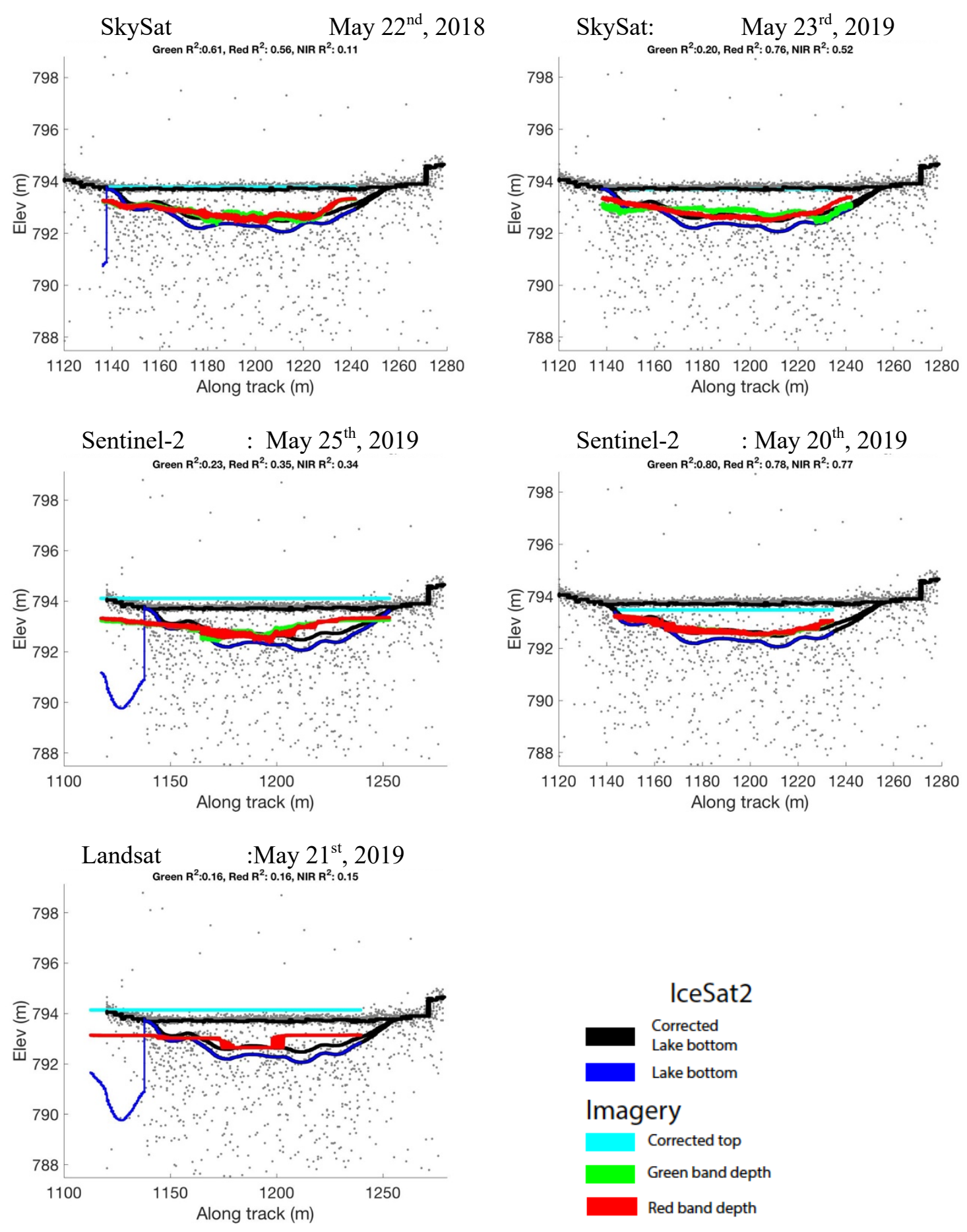

\section{IceSat2}

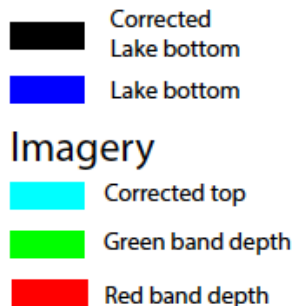




\section{Lake 2:}
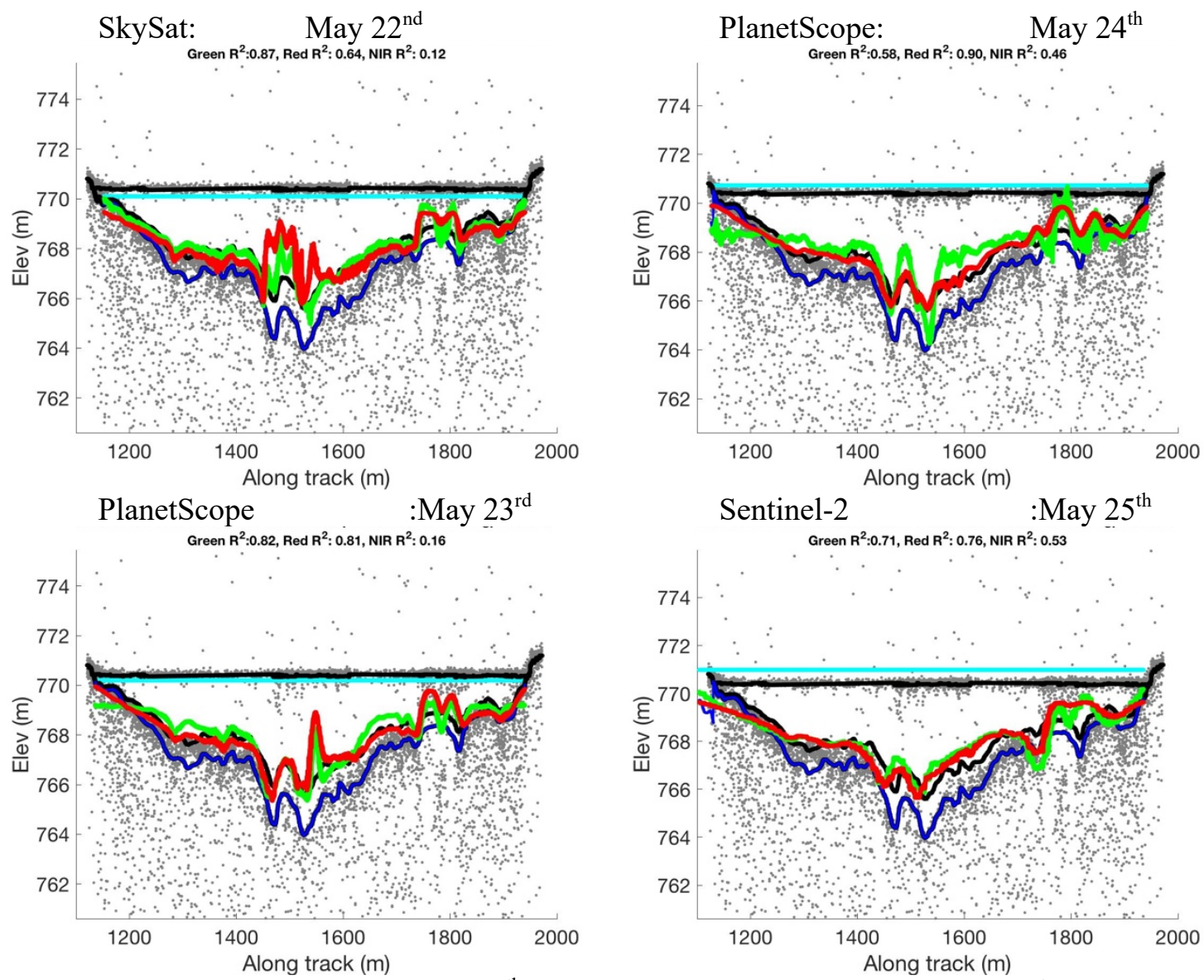

Sentinel-2

:May $25^{\text {th }}$

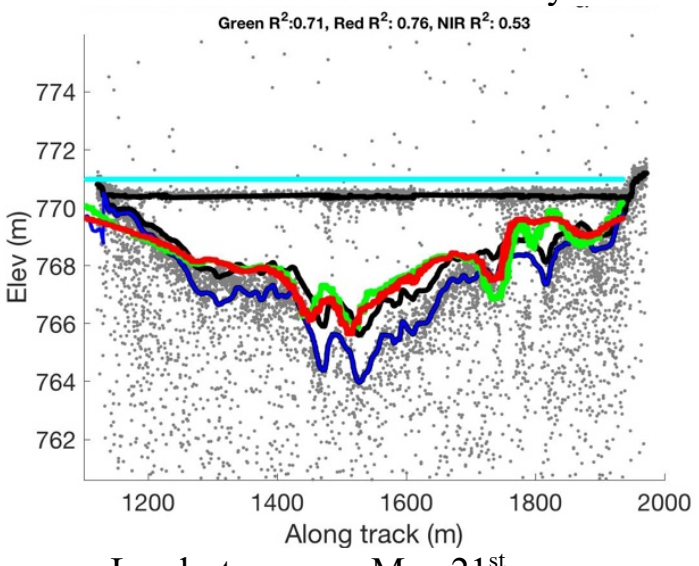

Sentinel-2

May $20^{\text {th }}$
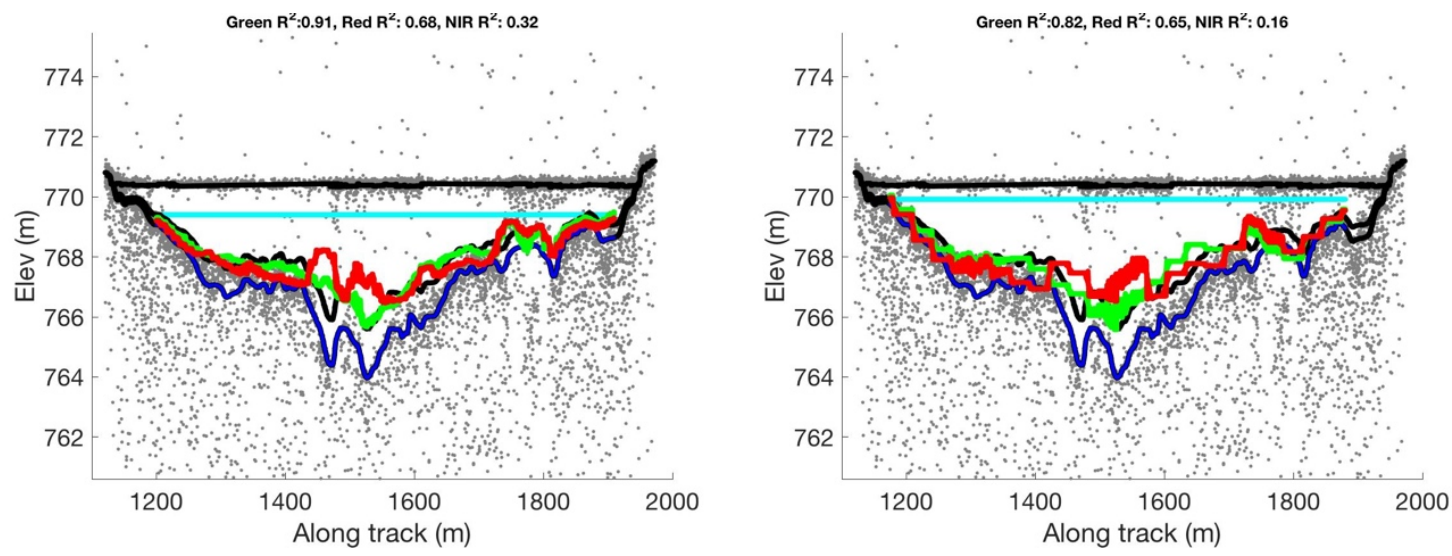
Lake 3:

SkySat:

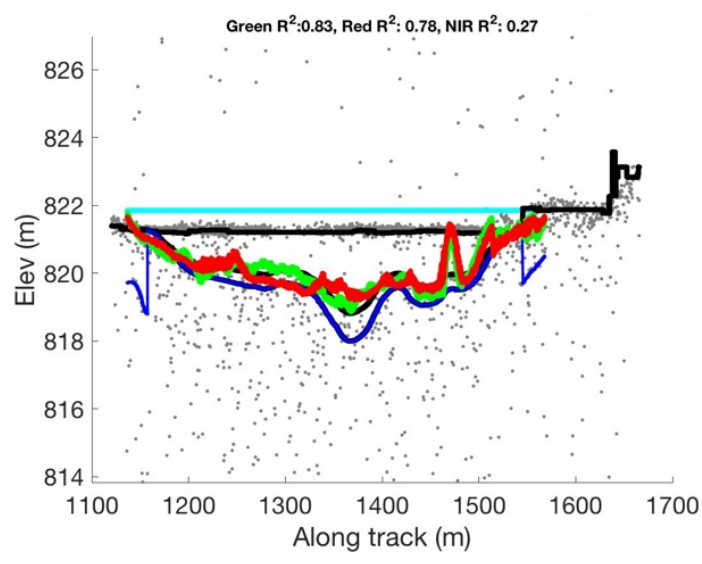

Sentinel-2

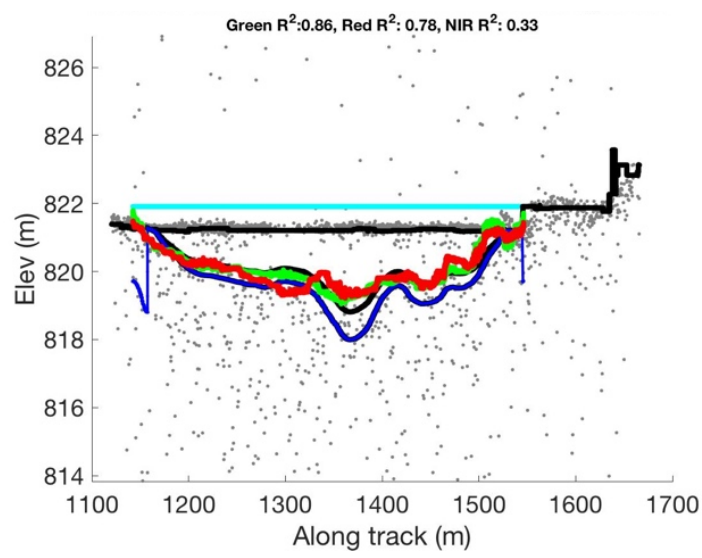

Sentinel-2

May $25^{\text {th }}$

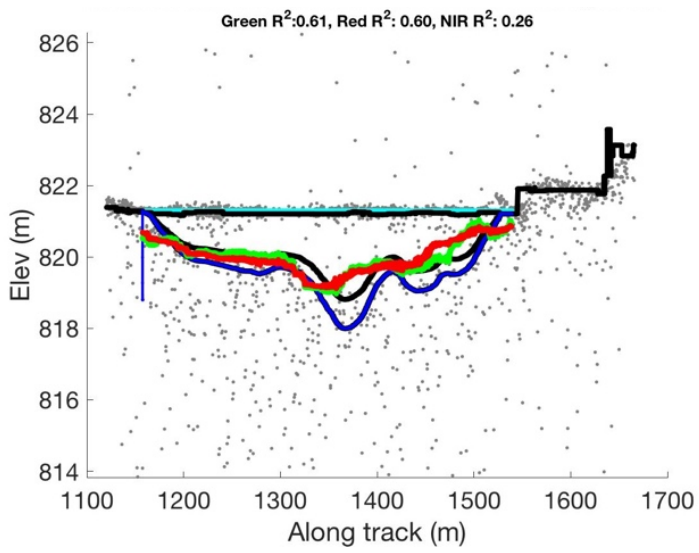

Landsat $\quad$ May $21^{\text {st }}$

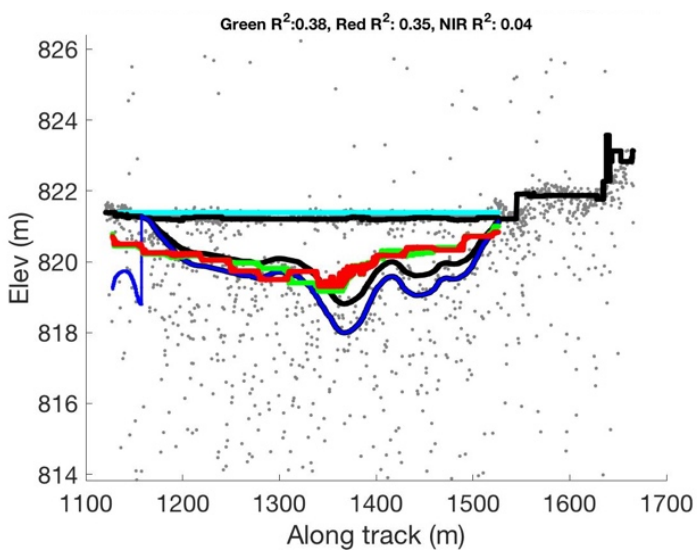

IceSat2

Corrected

Lake bottom

Lake bottom

Imagery

Corrected top

Green band depth

Red band depth 


\section{Lake 4:}

SkySat May 22 $2^{\text {nd }}$

PlanetScope

May $24^{\text {th }}$
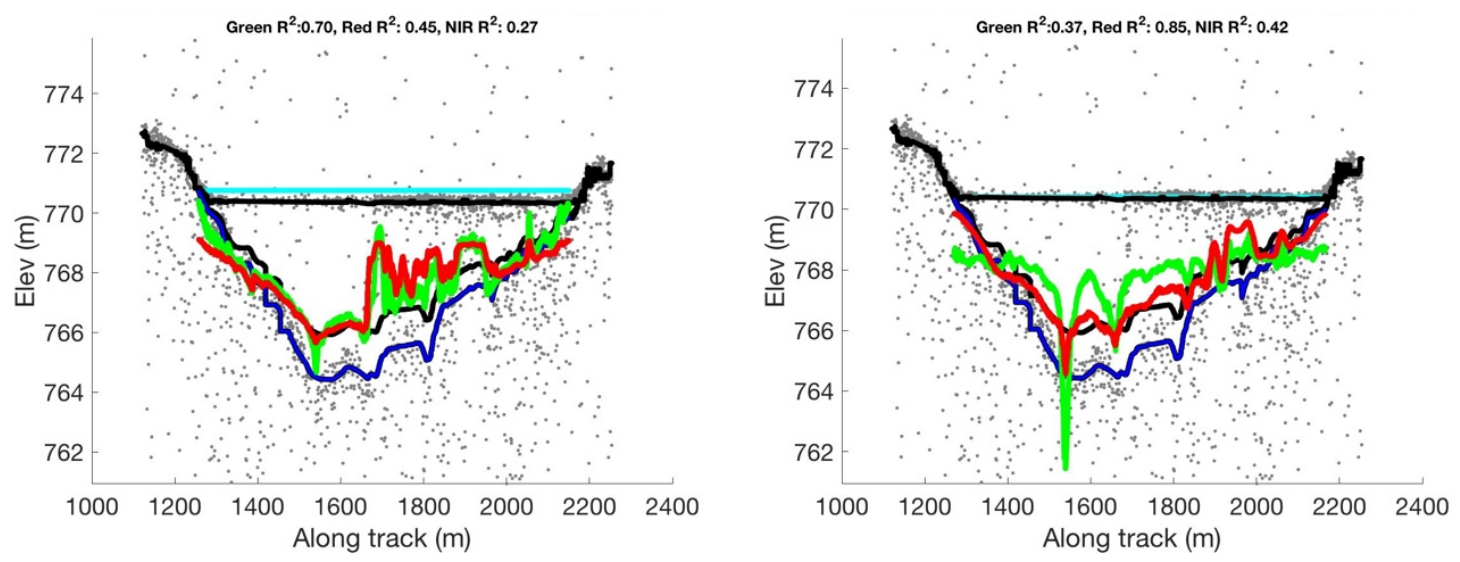

PlanetScope

May $23^{\text {rd }}$

Sentinel-2

May $25^{\text {th }}$
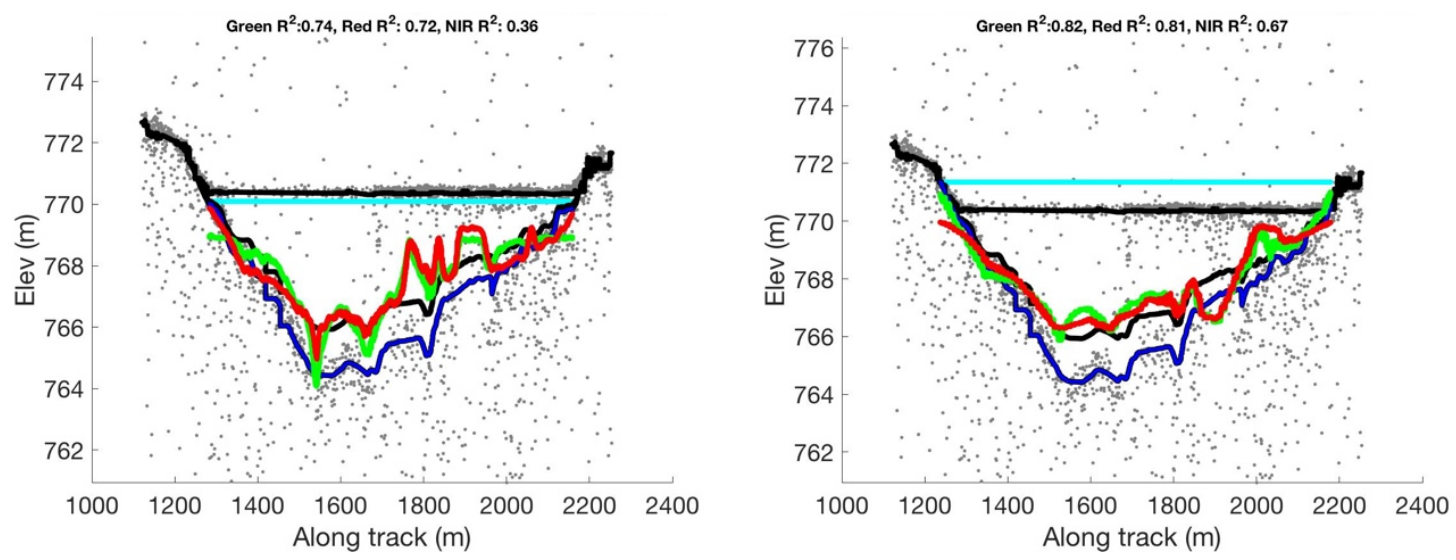

Sentinel-2 May 20

Landsat May $21^{\text {st }}$
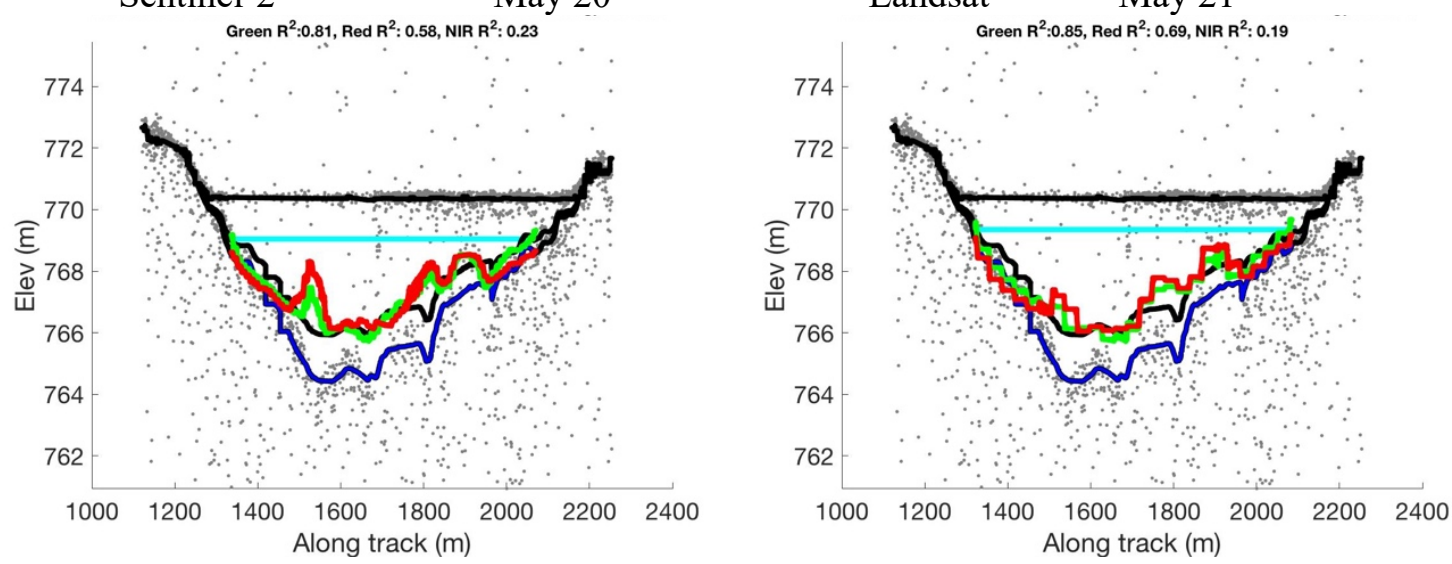


\section{Lake 5:}
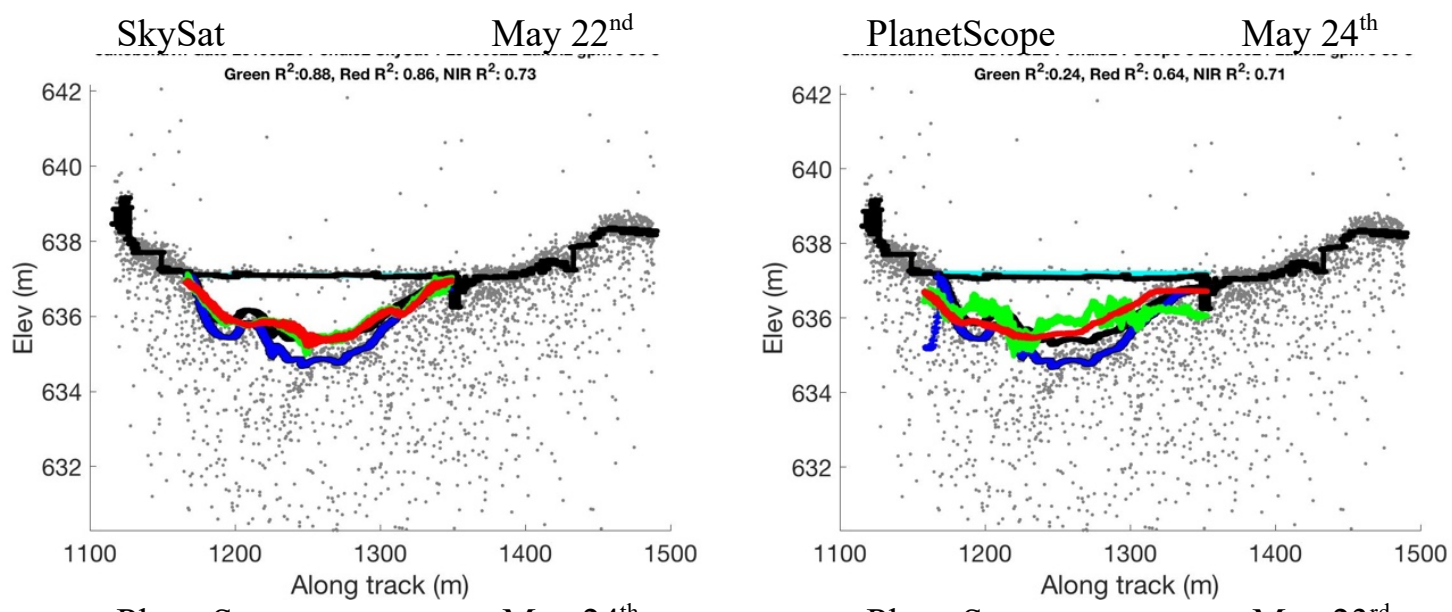

PlanetScope

:May $24^{\text {th }}$
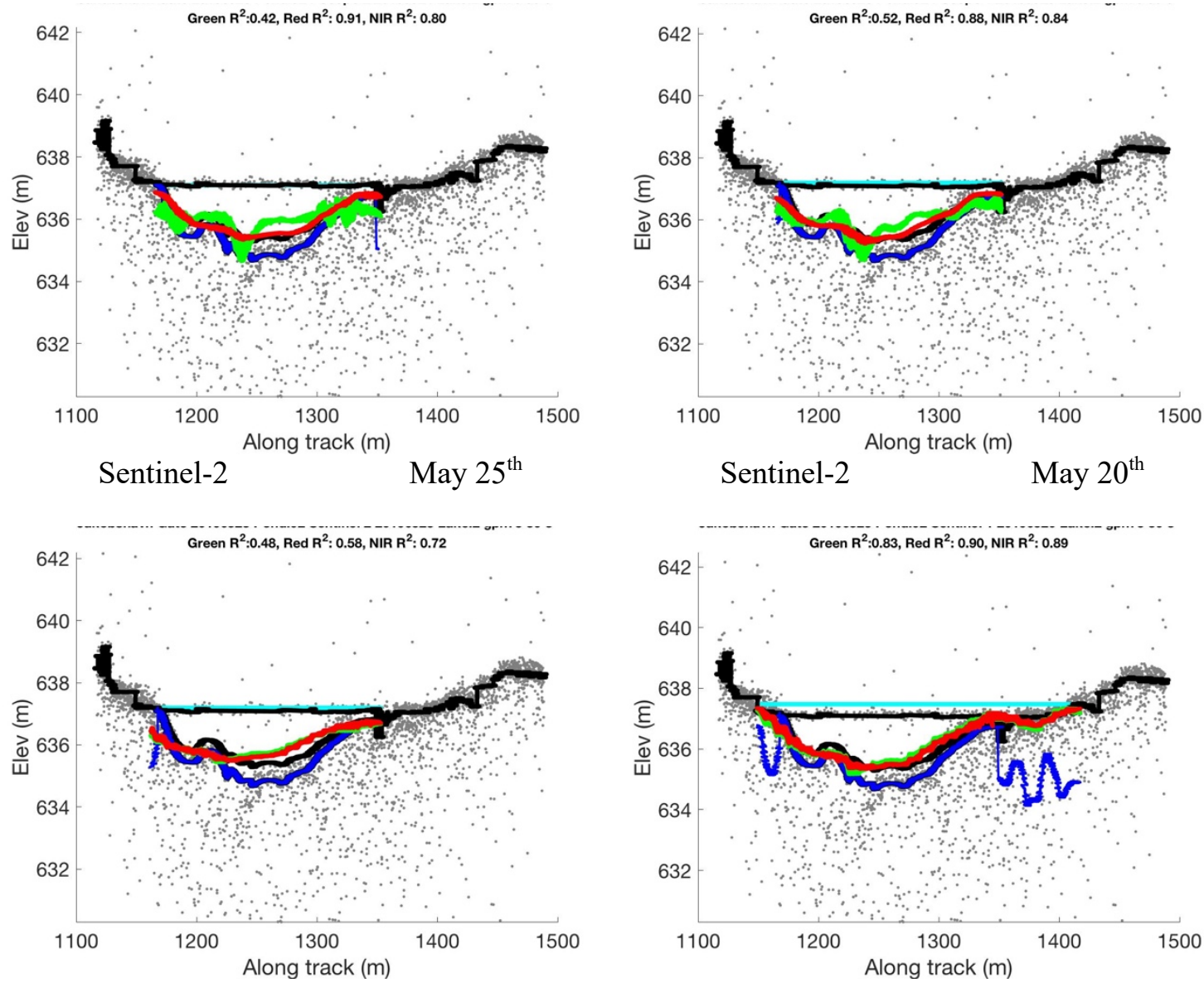


\section{Lake $5 \quad$ cont...}

Landsat

May $23^{\text {rd }}$

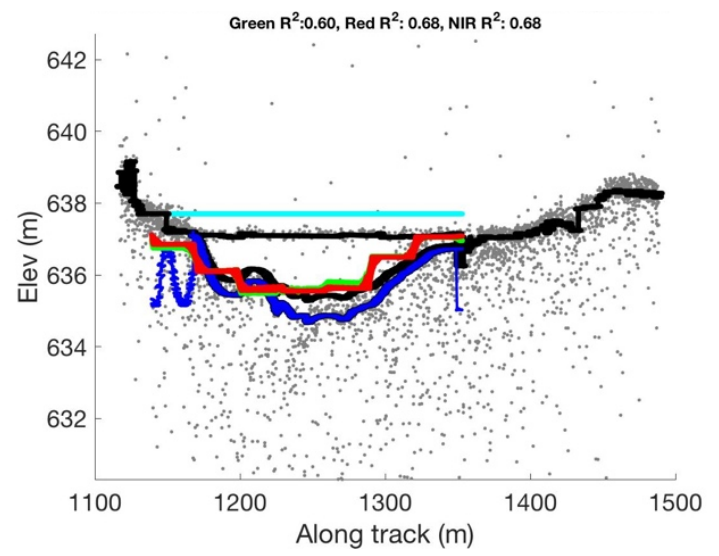

\section{IceSat2}

Corrected

Lake bottom

Lake bottom

Imagery

Corrected top

Green band depth

Red band depth 
Lake 1

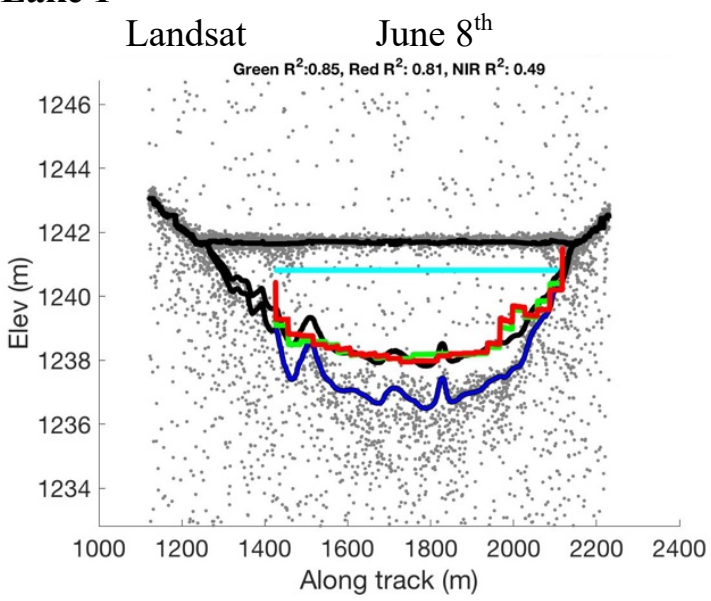

\section{Lake 2:}

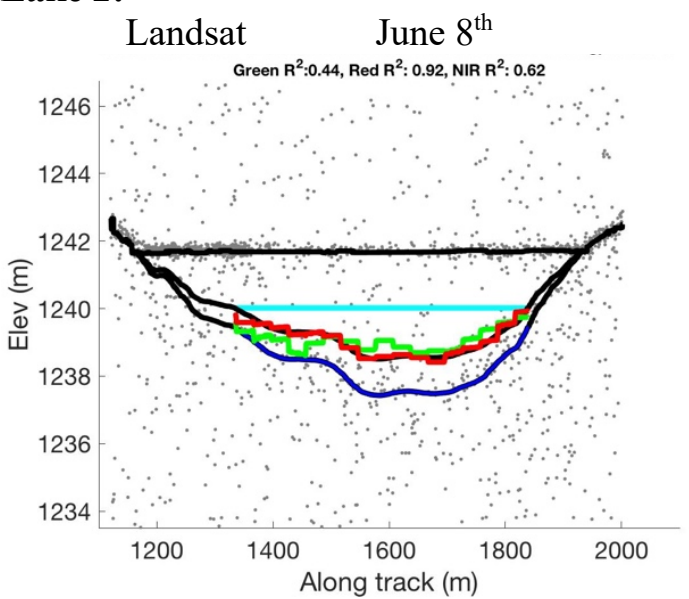

\section{Lake 3:}

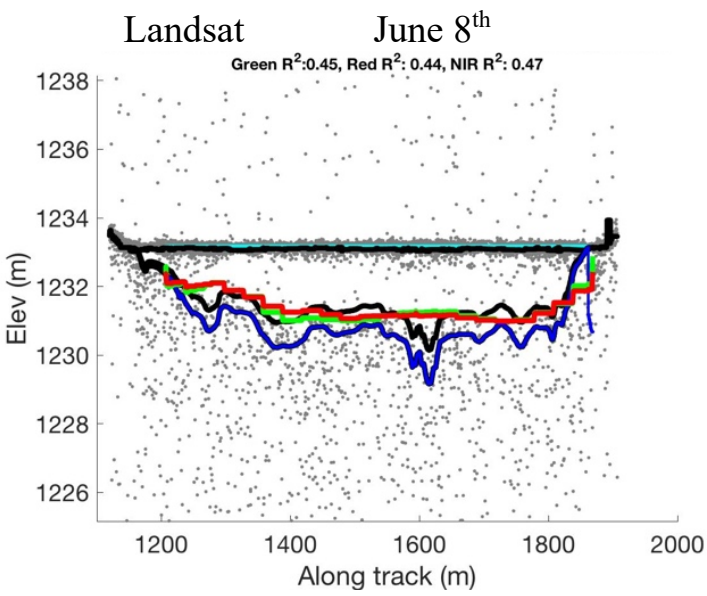

\section{IceSat2}

Corrected Lake bottom

Lake bottom Imagery

Corrected top

Green band depth

Red band depth

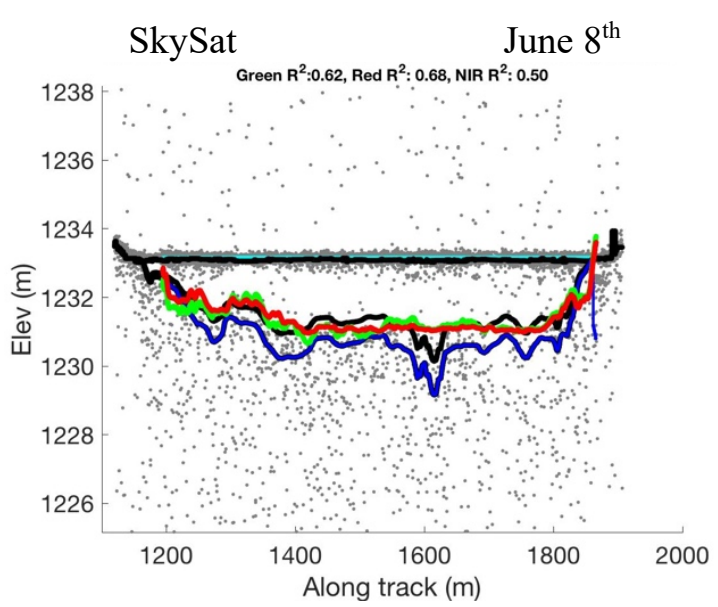


RGT 1108

June 9th, 2019

\section{Lake 4}

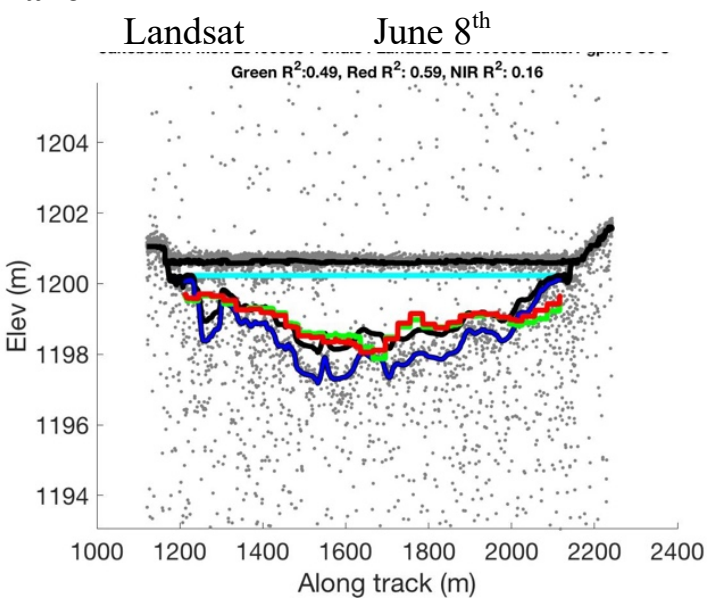

Lake 5

Landsat

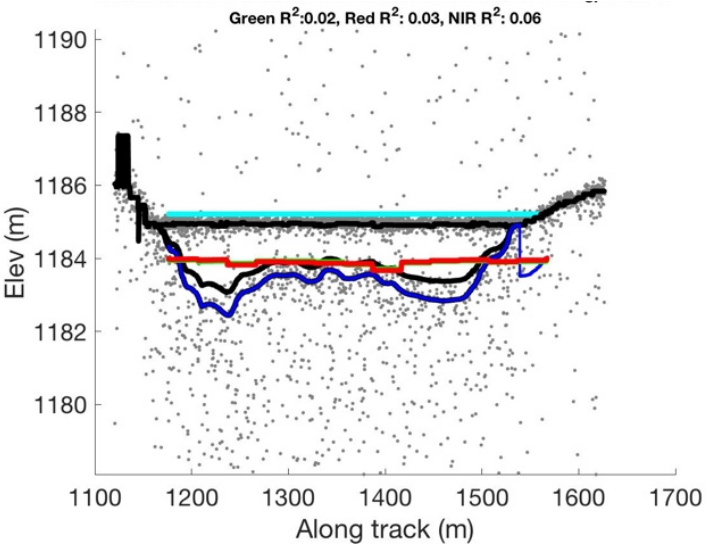

Lake 6

Landsat

June $8^{\text {th }}$

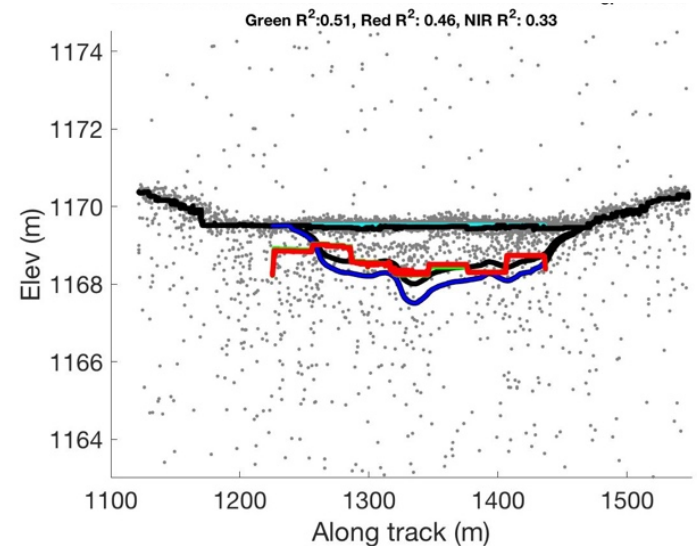

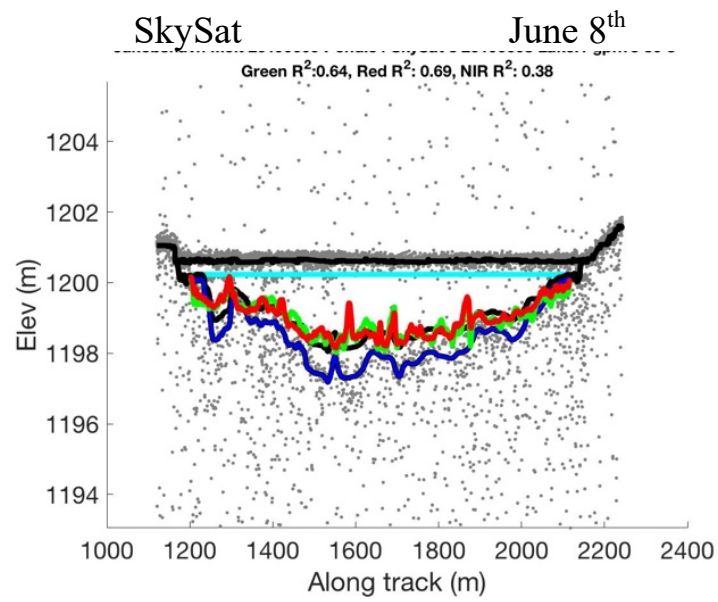

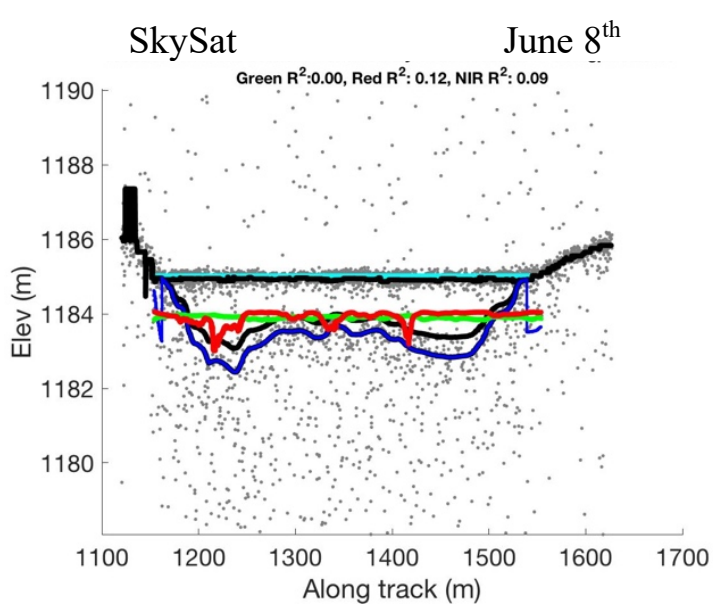

SkySat June $8^{\text {th }}$

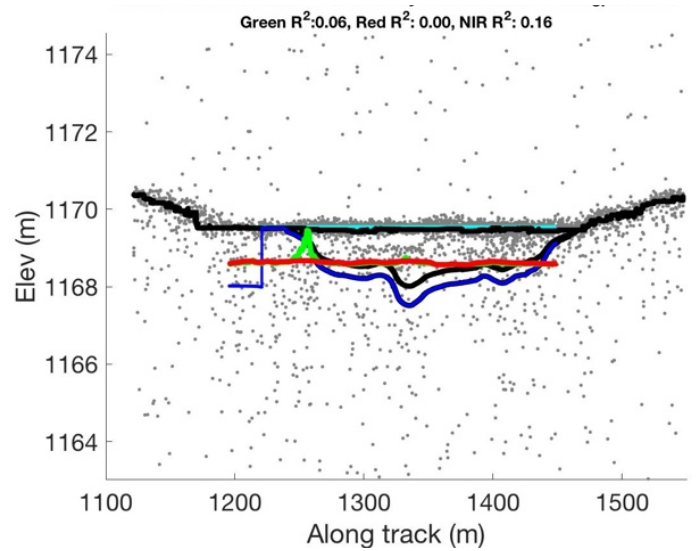




\section{Lake 7}

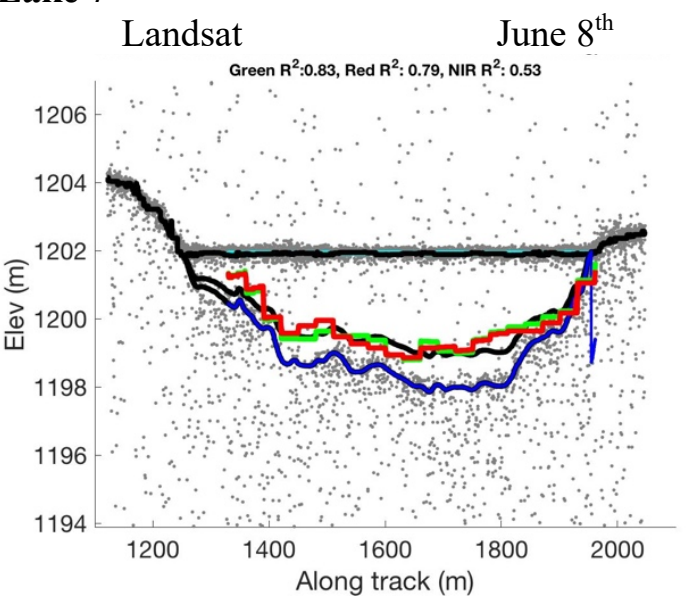

\section{Lake 8}

Landsat

June $8^{\text {th }}$

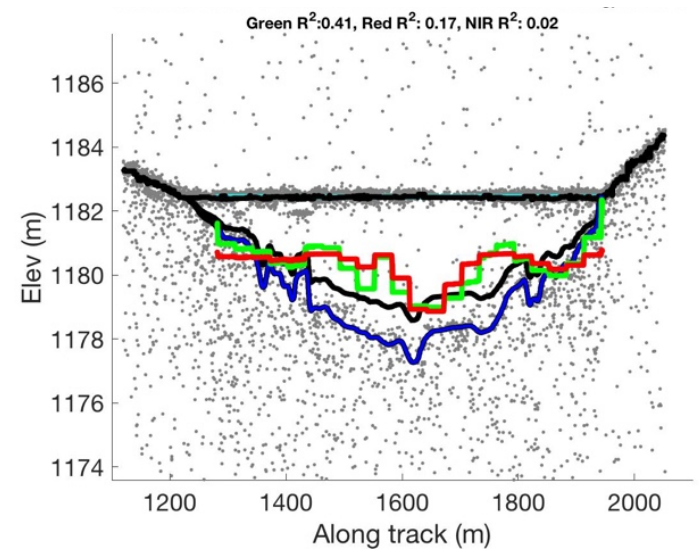

Lake 9

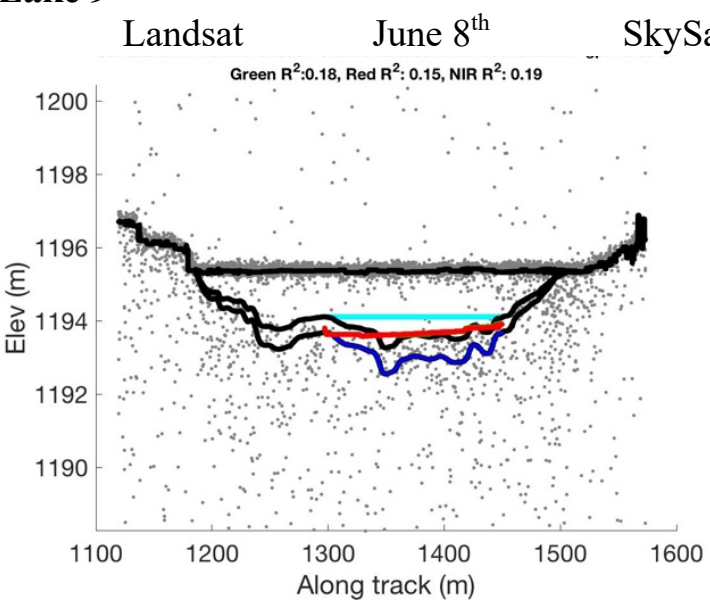

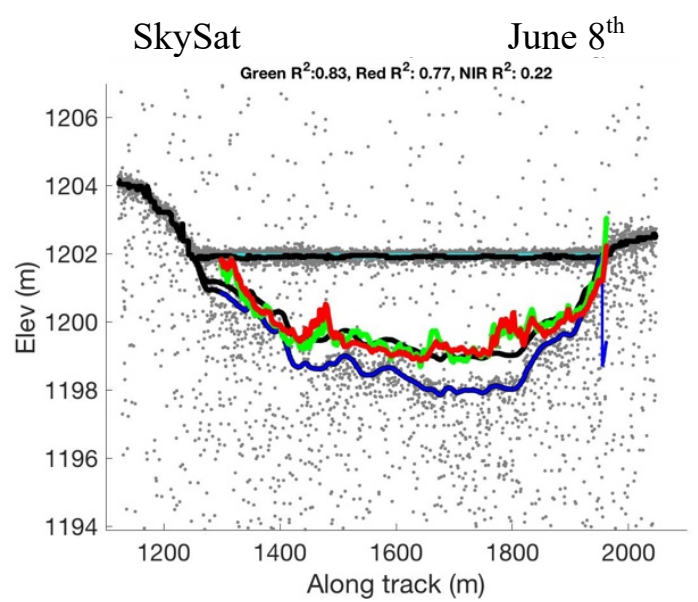




\section{Lake 10}
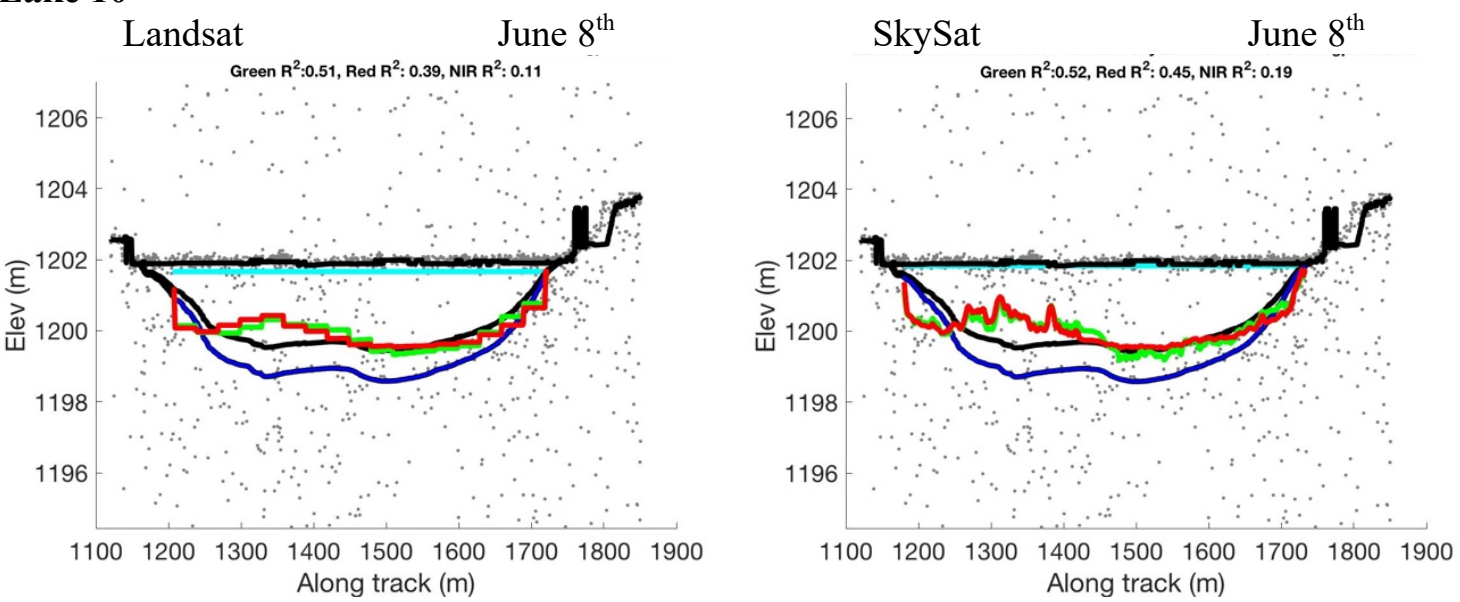

Lake 11

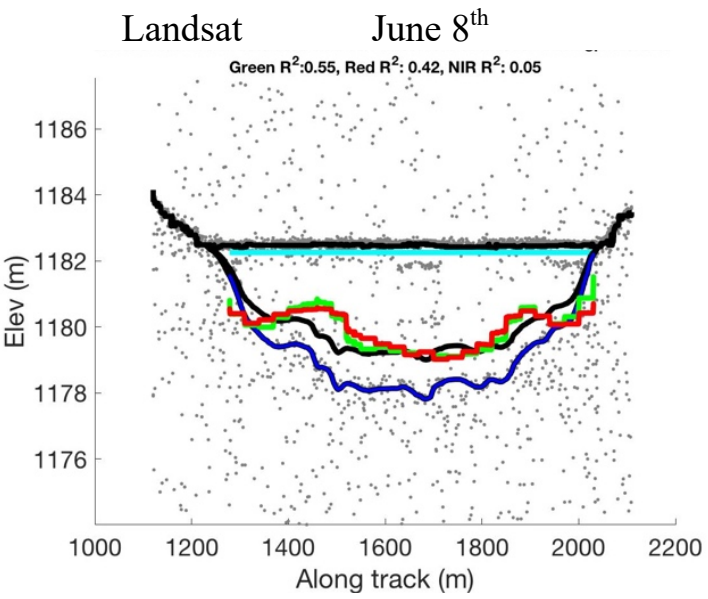




\section{Lake 1}

Landsat June $8^{\text {th }} \quad$ Sentinel-2 June $16^{\text {th }}$
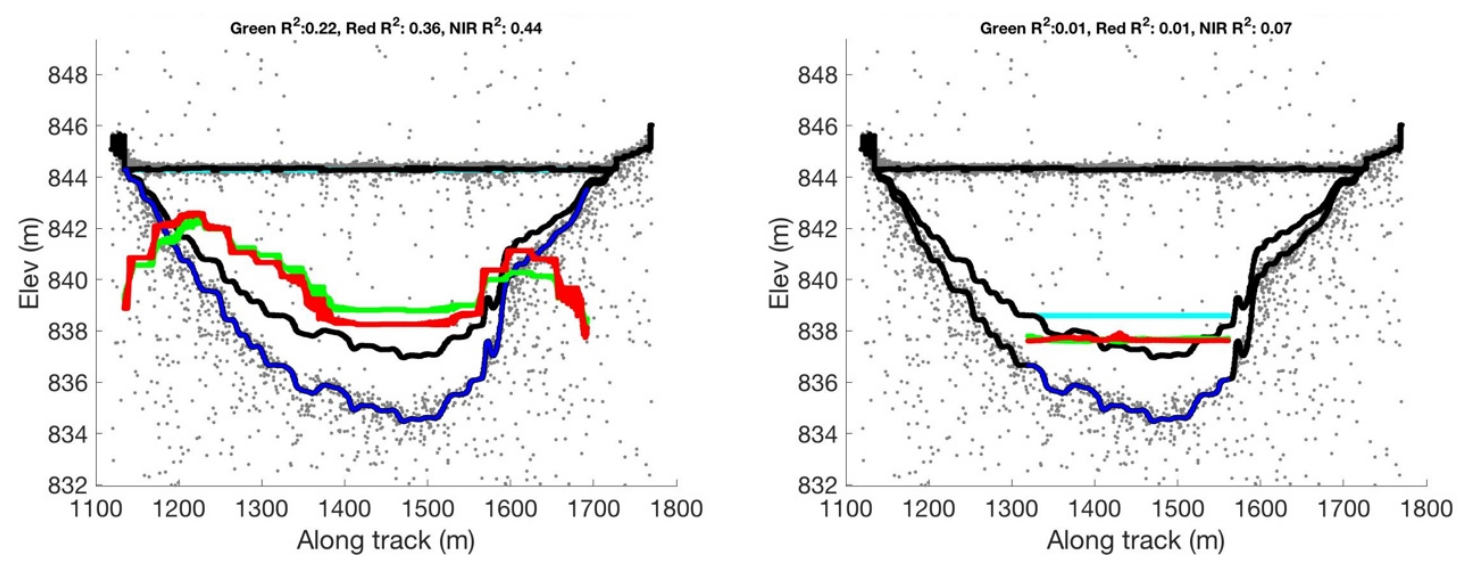

Lake 2

Sentinel-2 June $16^{\text {th }}$

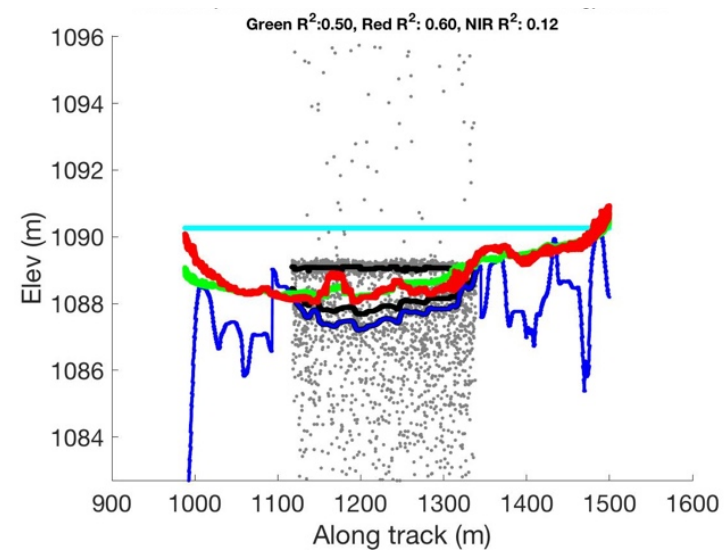




\section{Lake 3}
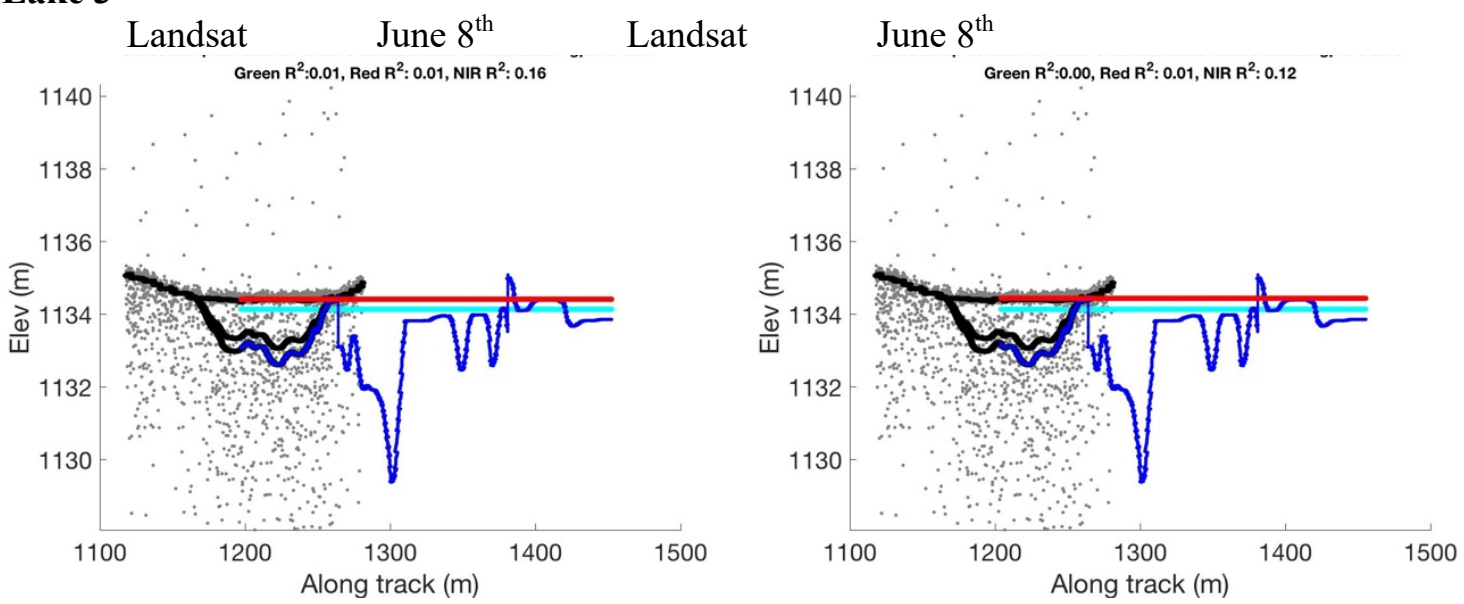

Sentinel

June $16^{\text {th }}$

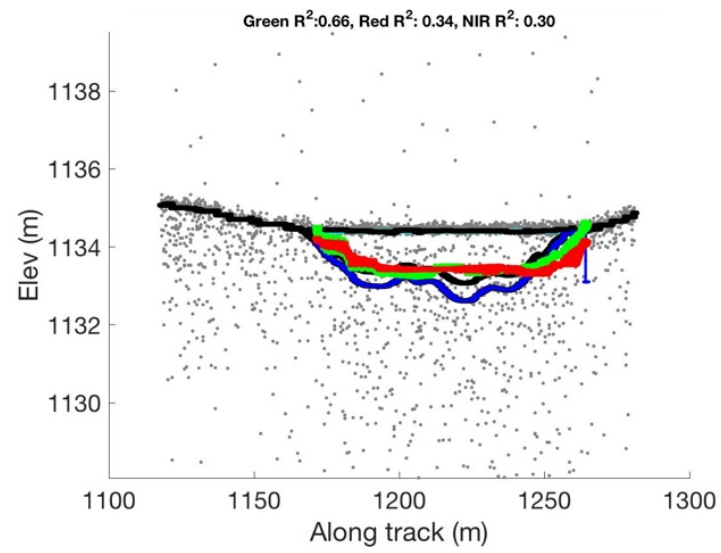

\section{Lake 4}

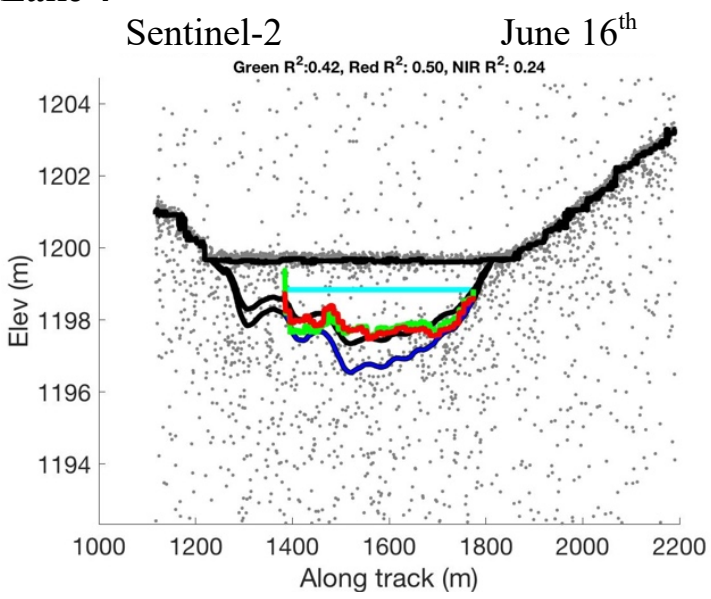




\section{Lake 5}

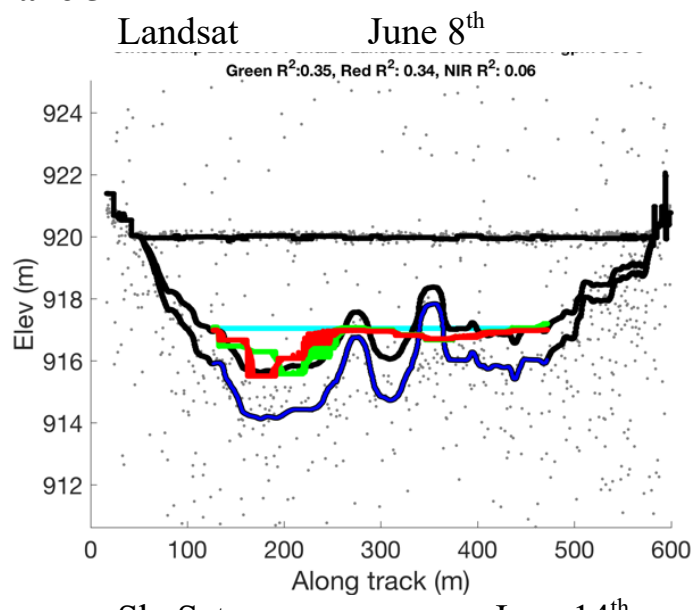

SkySat:

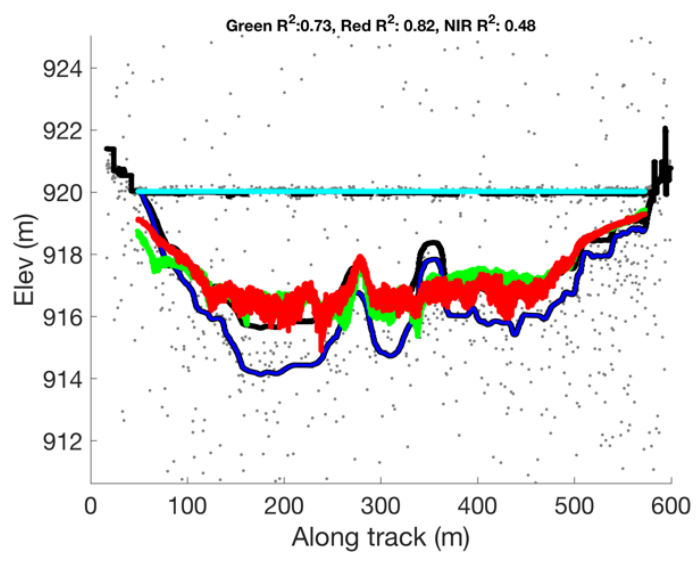

\section{Lake 6}

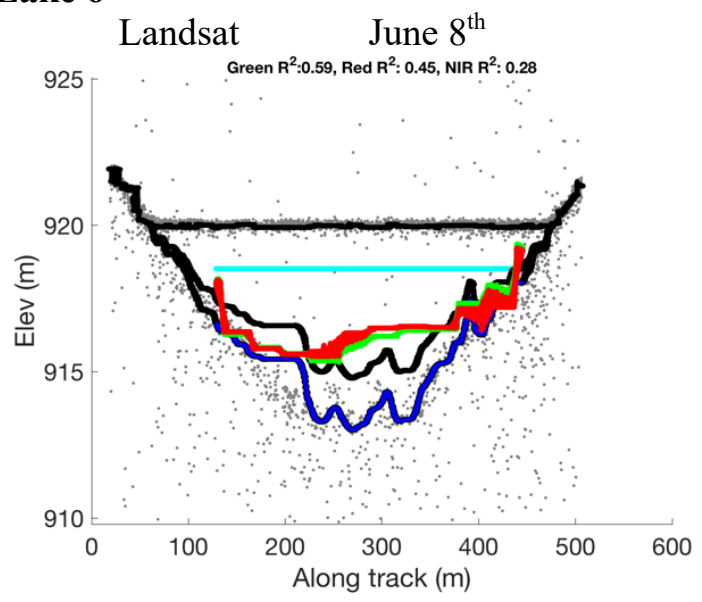

Sentinel-2

June $16^{\text {th }}$

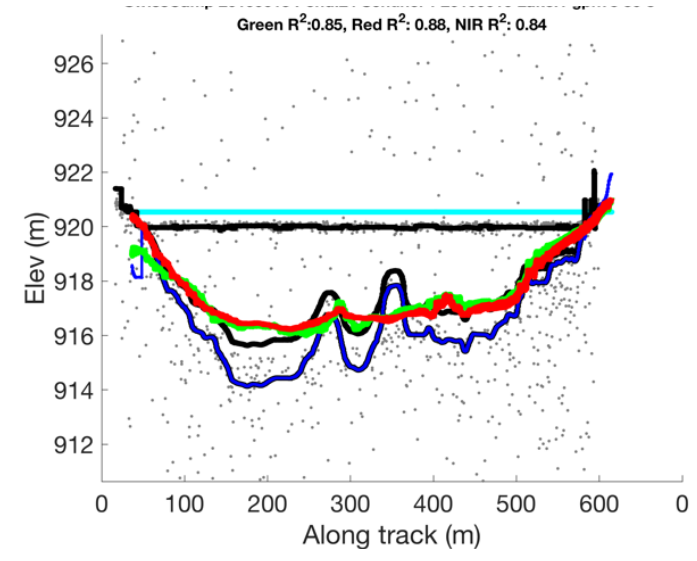




\section{Lake 6 cont...}

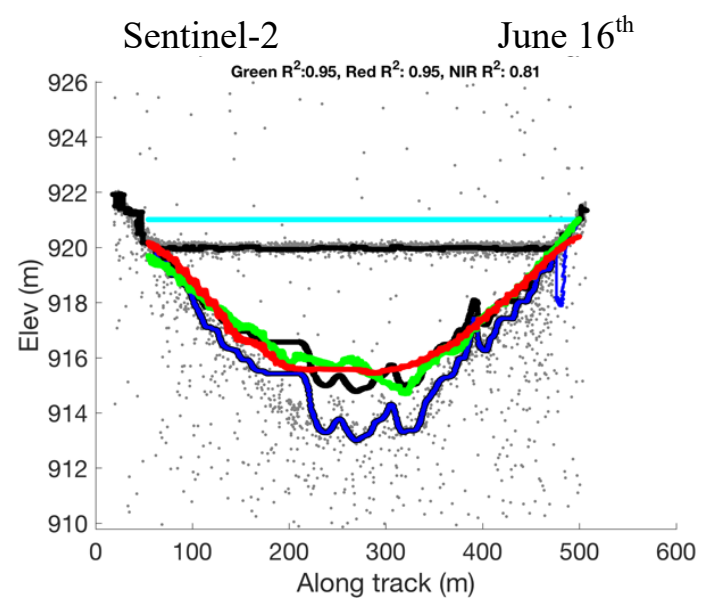

\section{Lake 7}

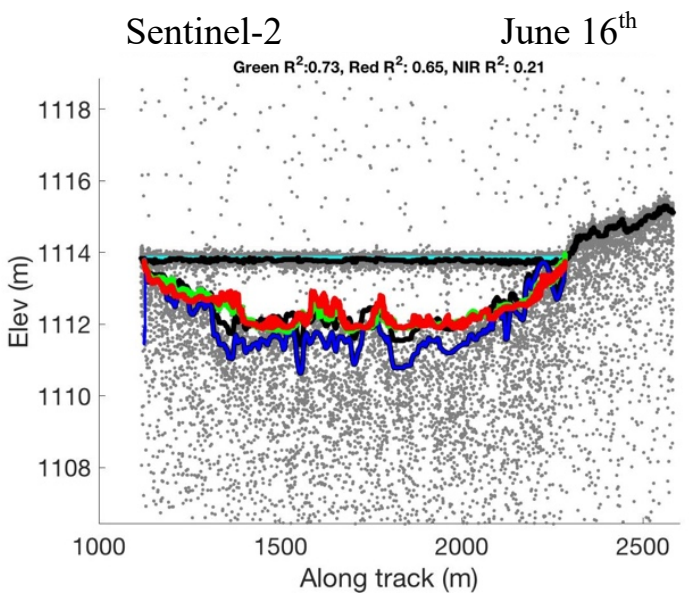

\section{Lake 8}

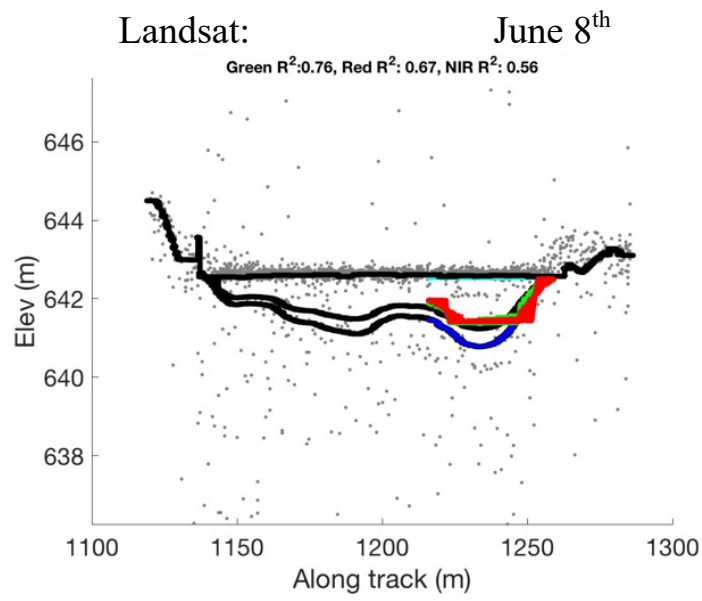

Sentinel-2 June $19^{\text {th }}$

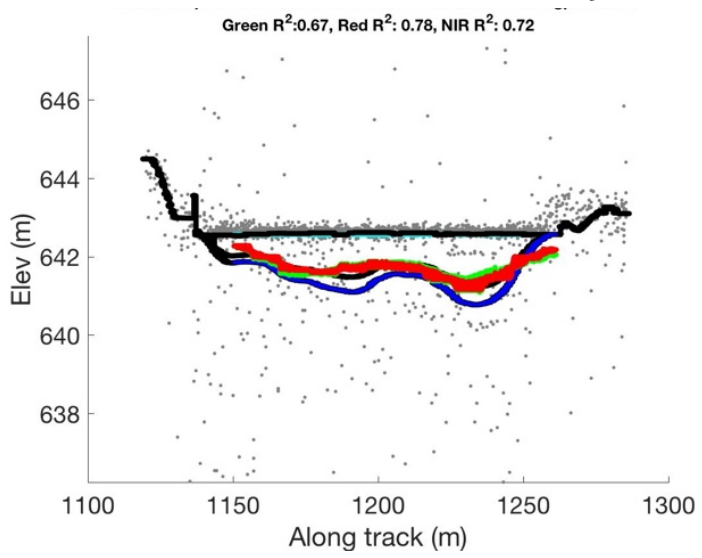




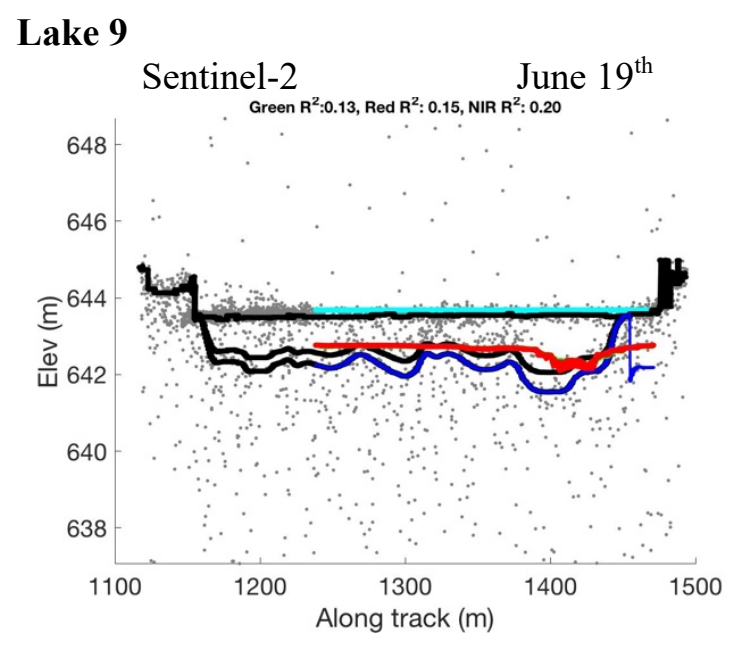




\section{Lake 1}

Landsat June $17^{\text {th }}$

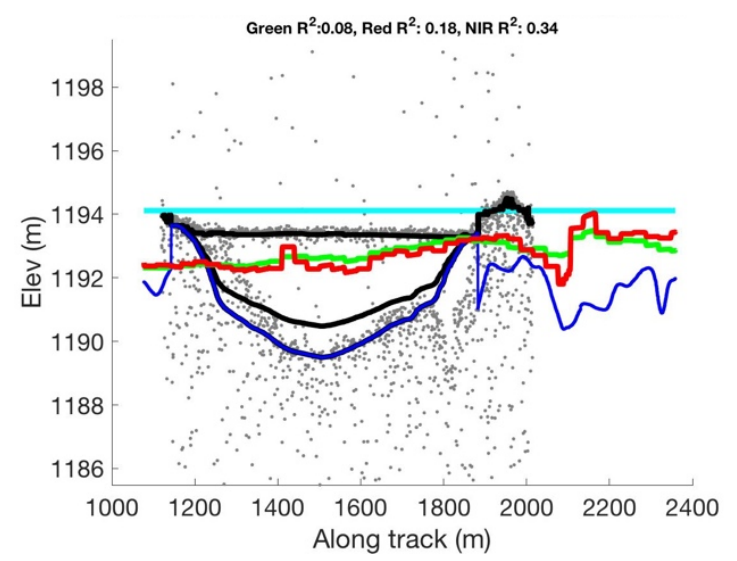

\section{Lake 2}

Landsat June $17^{\text {th }}$

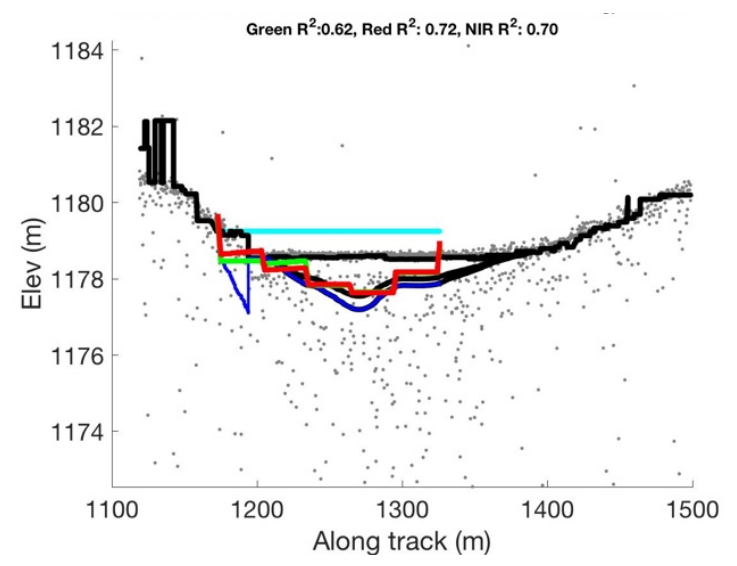

\section{Lake 3}

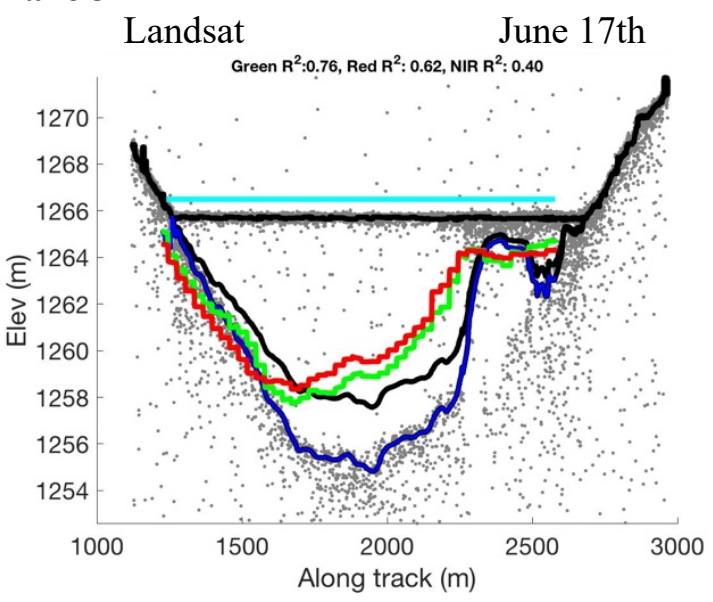




\section{Lake 4}

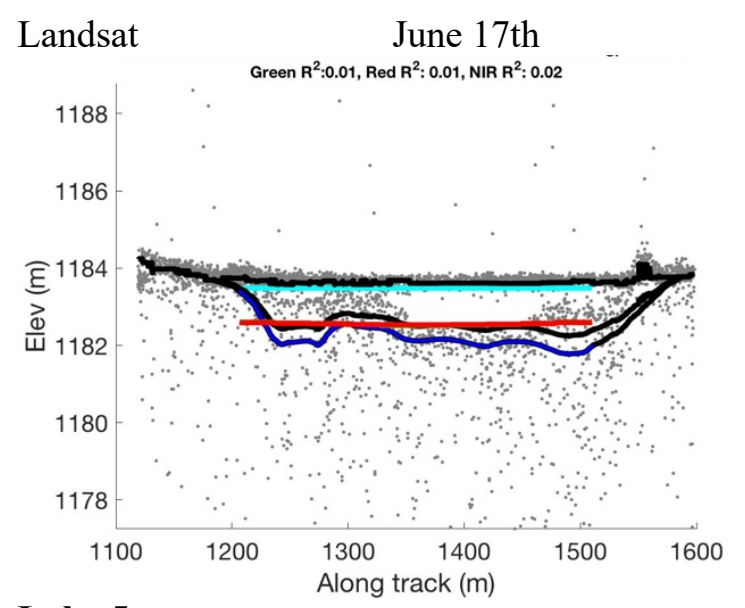

\section{Lake 5:}

Landsat June 17th

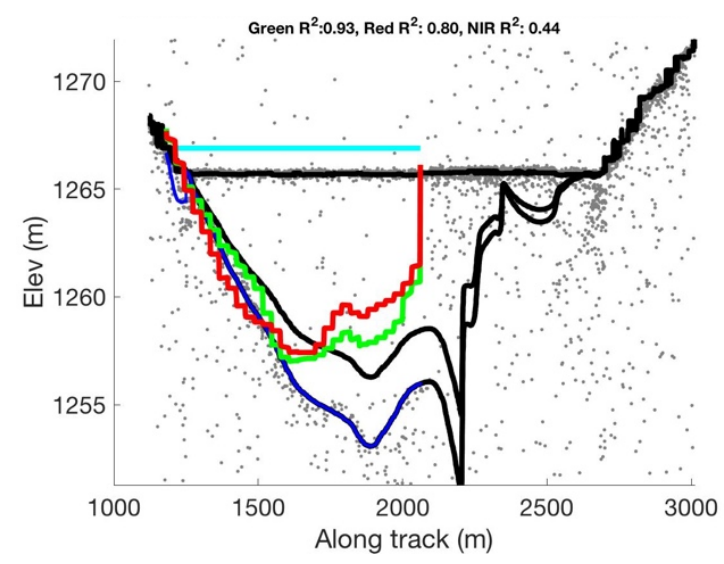

\section{Lake 6}

Landsat

June $17^{\text {th }}$

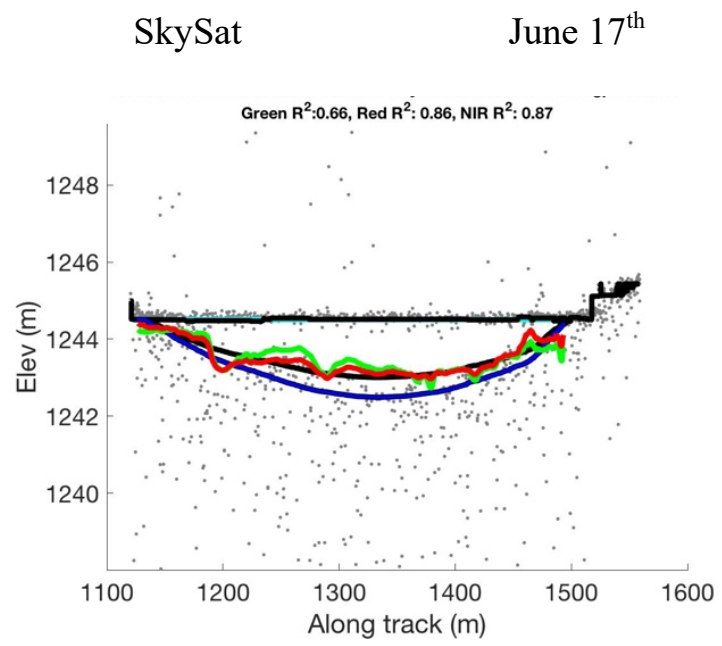




\section{Lake 7:}

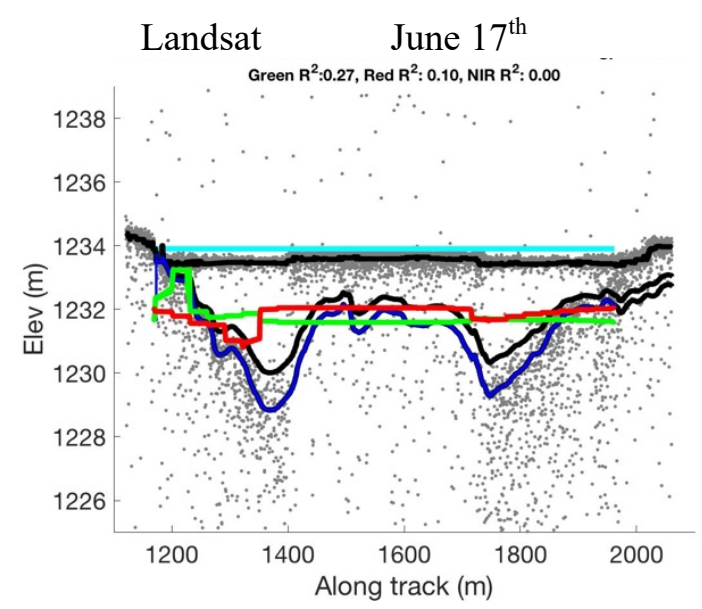

\section{Lake 8:}

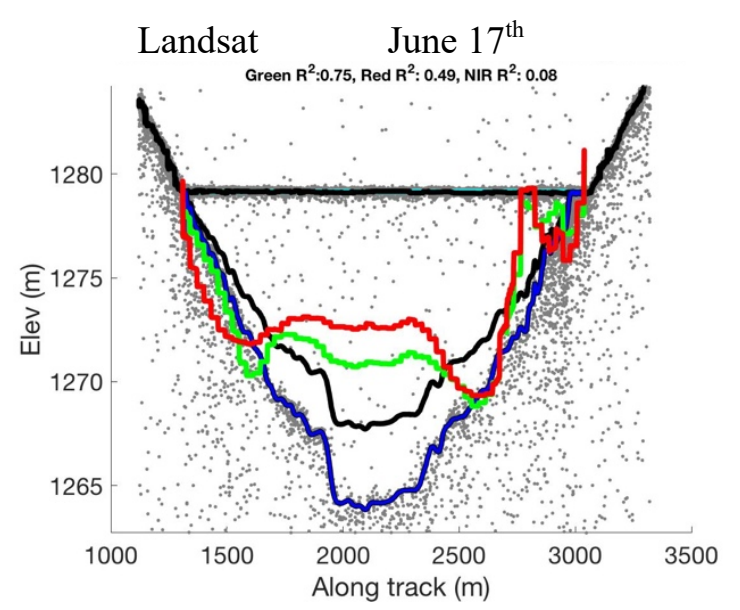

Lake 9:

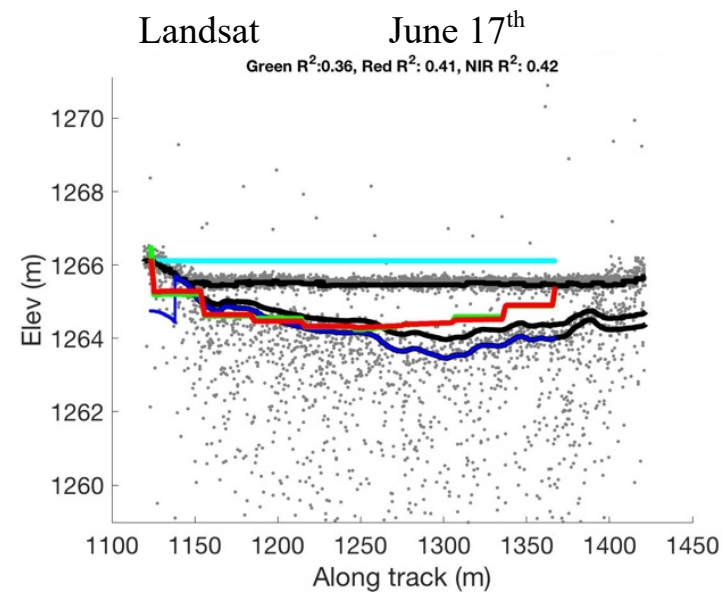




\section{Lake 10}

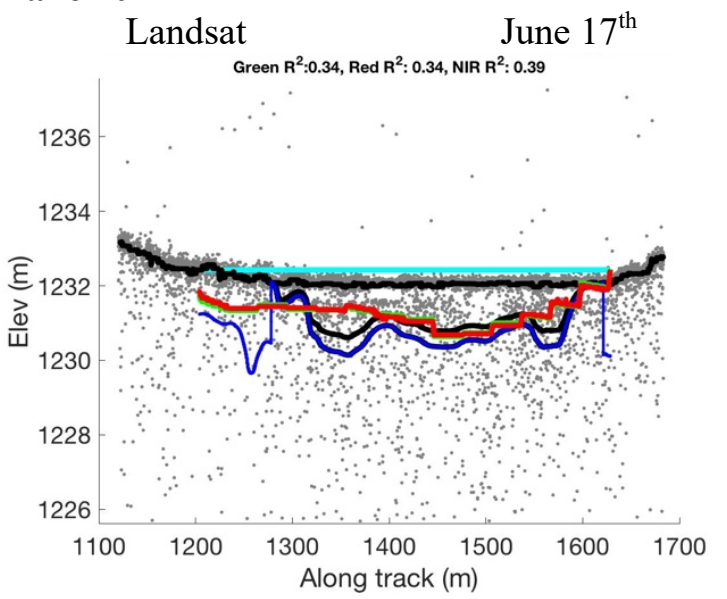

\section{Lake 11}

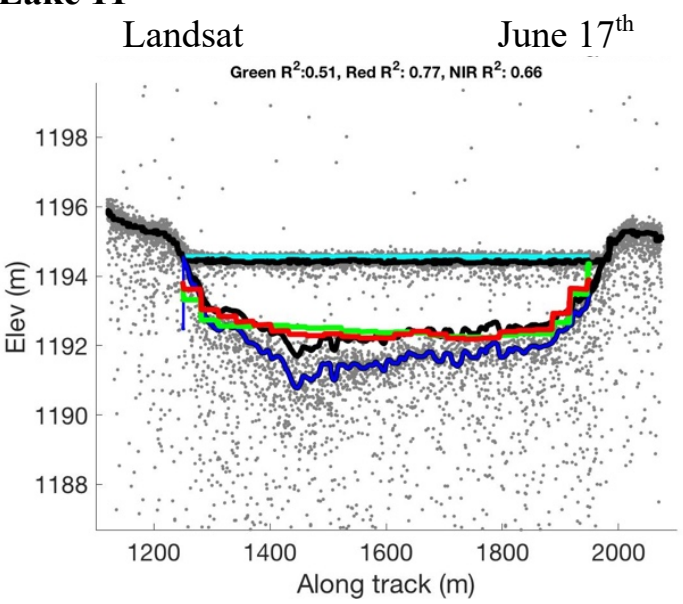

\section{Lake 12}

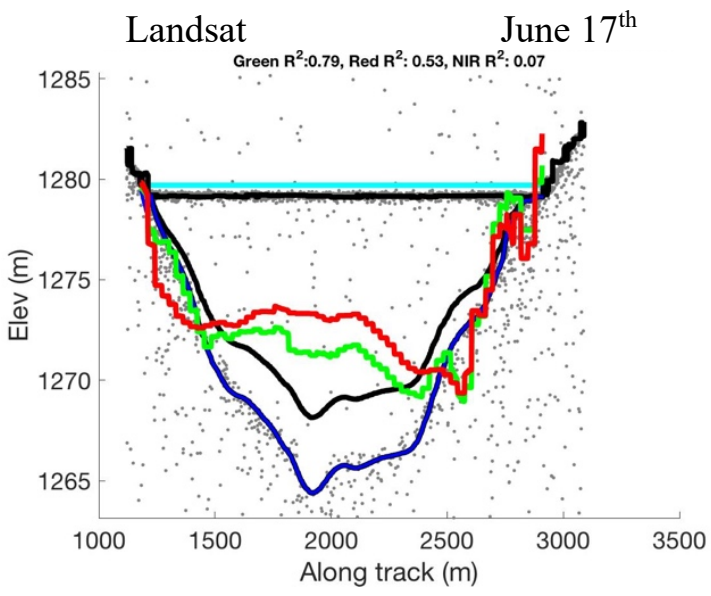

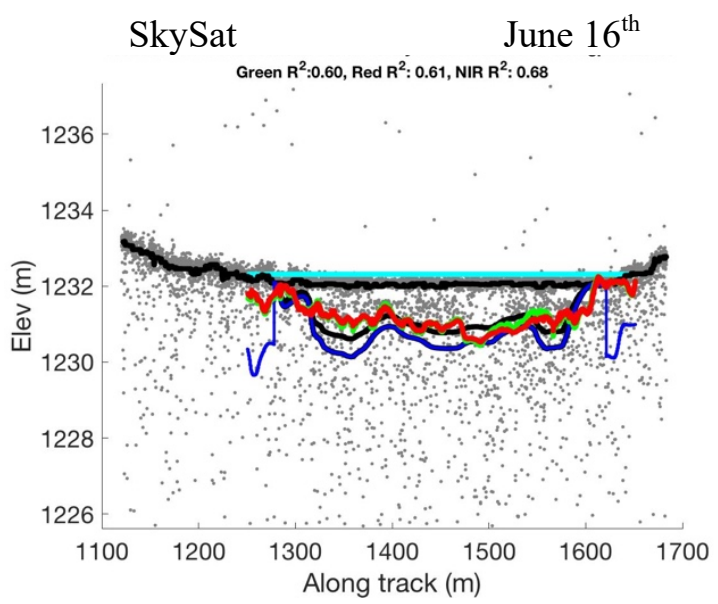




\section{Lake 13}

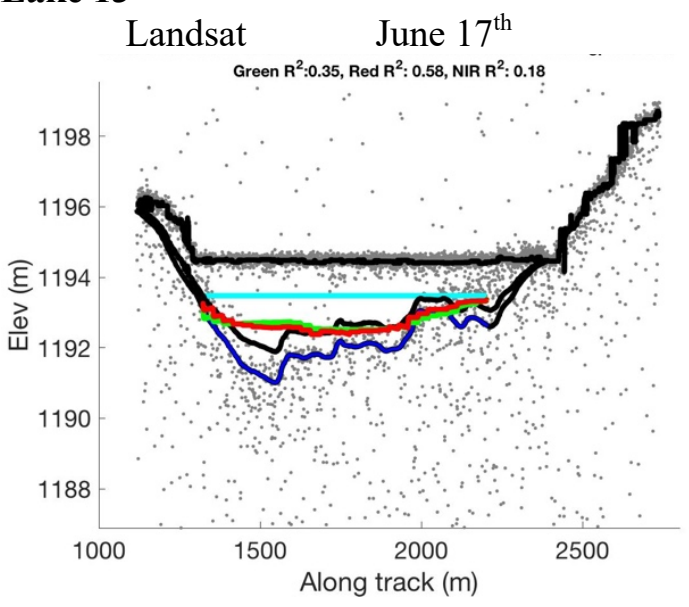

\section{Lake 14}

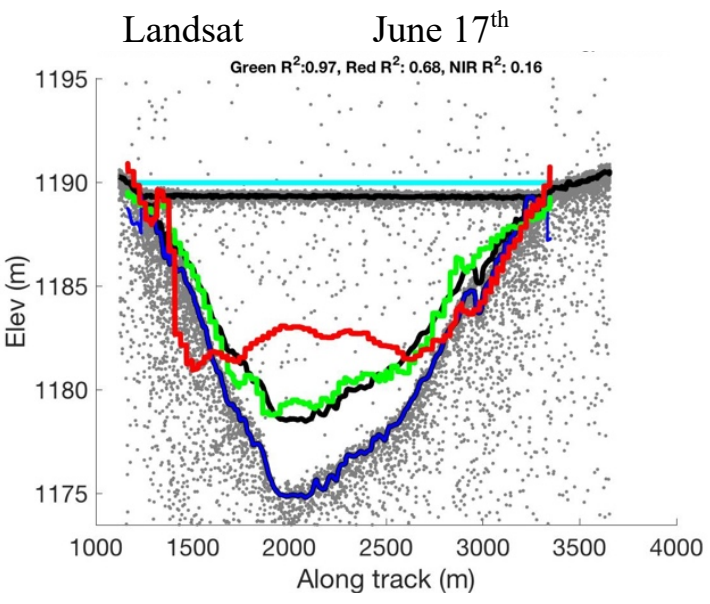

\section{Lake 15}

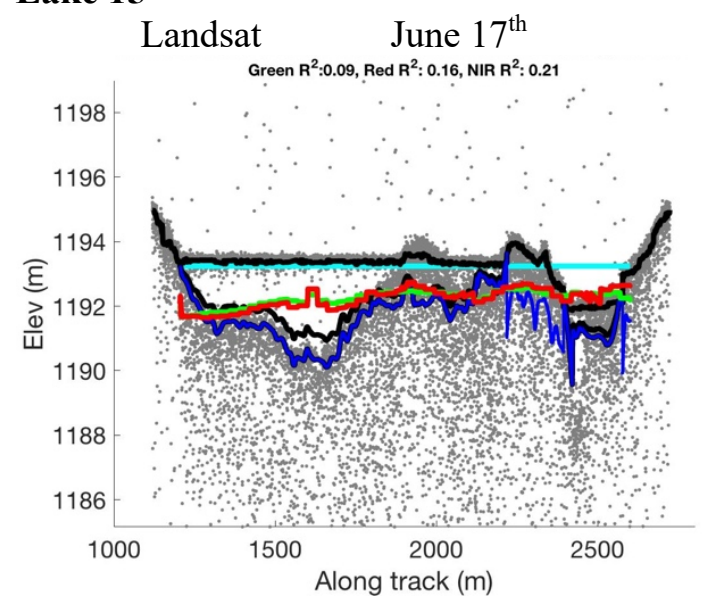




\section{Lake 16}

Landsat June $17^{\text {th }}$

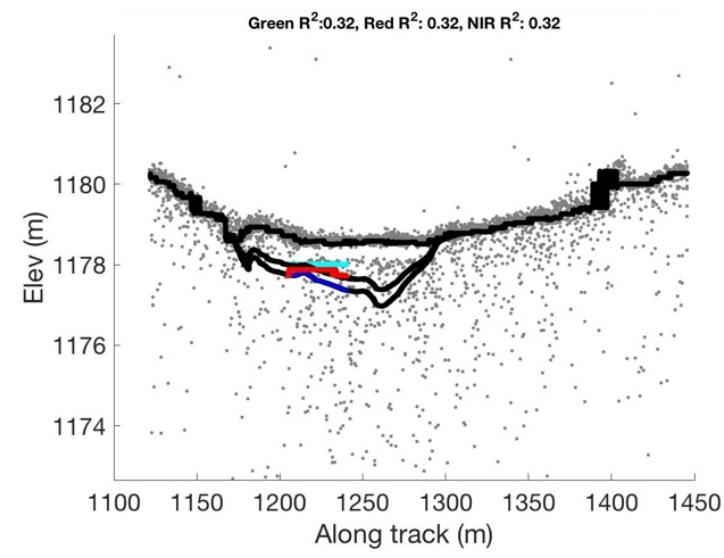

\section{Lake 17}

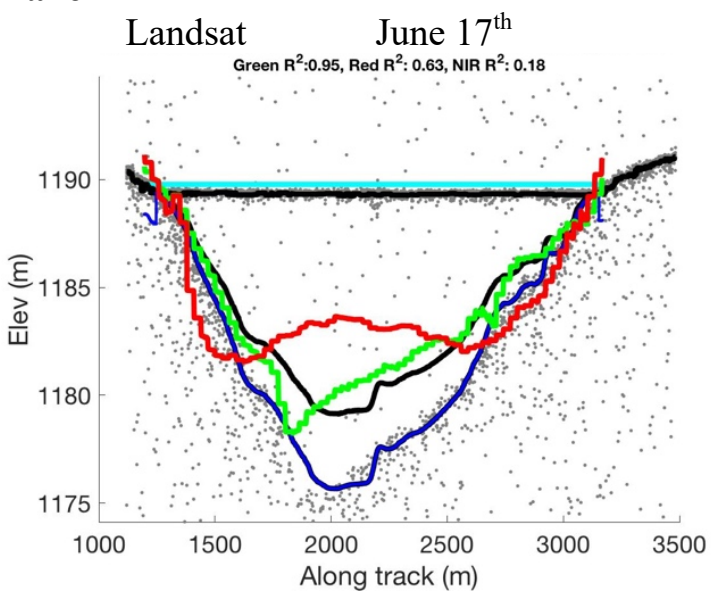

Lake 18

Sentinel-2

June $16^{\text {th }}$

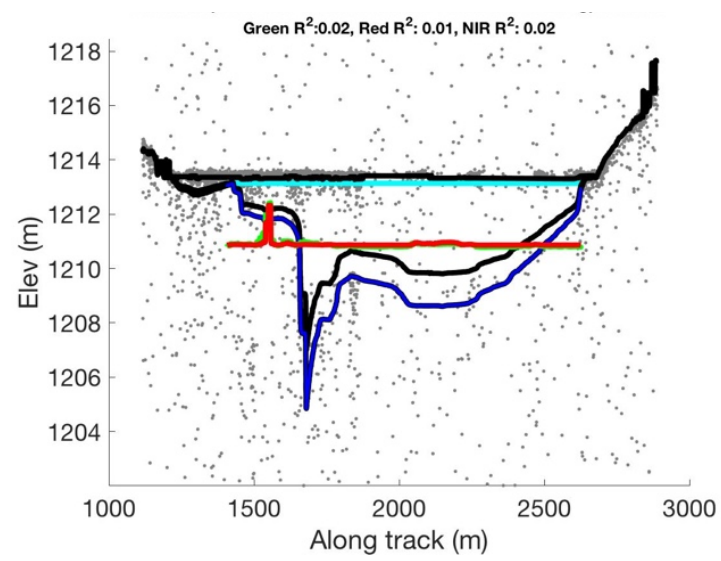




\section{Lake 19}

Sentinel-2 June $16^{\text {th }}$
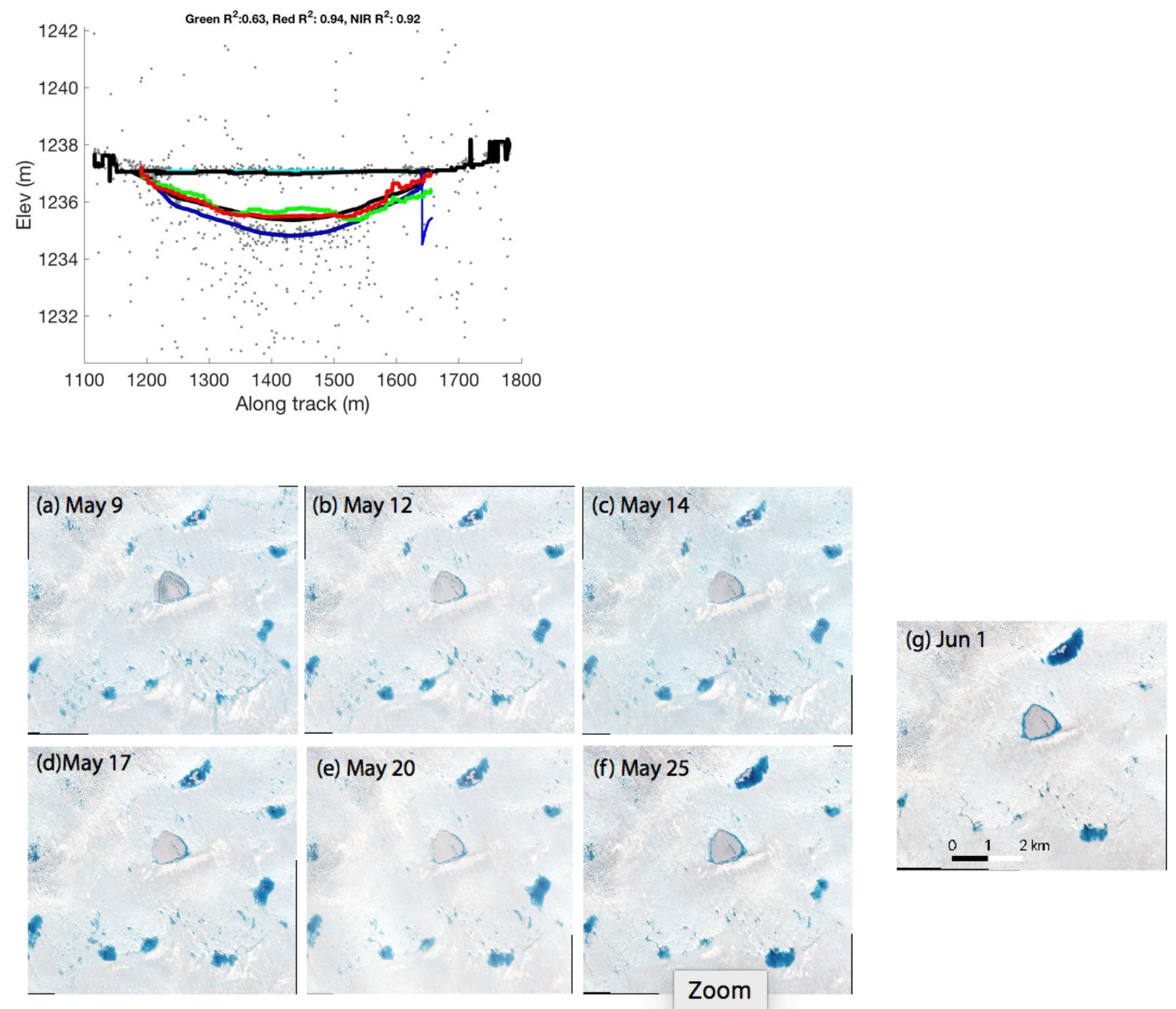

Figure S4: Lake evolution throughout the season in region of Western Greenland (see Fig. 1 in main) from Sentinel-2 imagery. Surface water extent (where NDWIice > 0.2) remains consistent at 3\% 

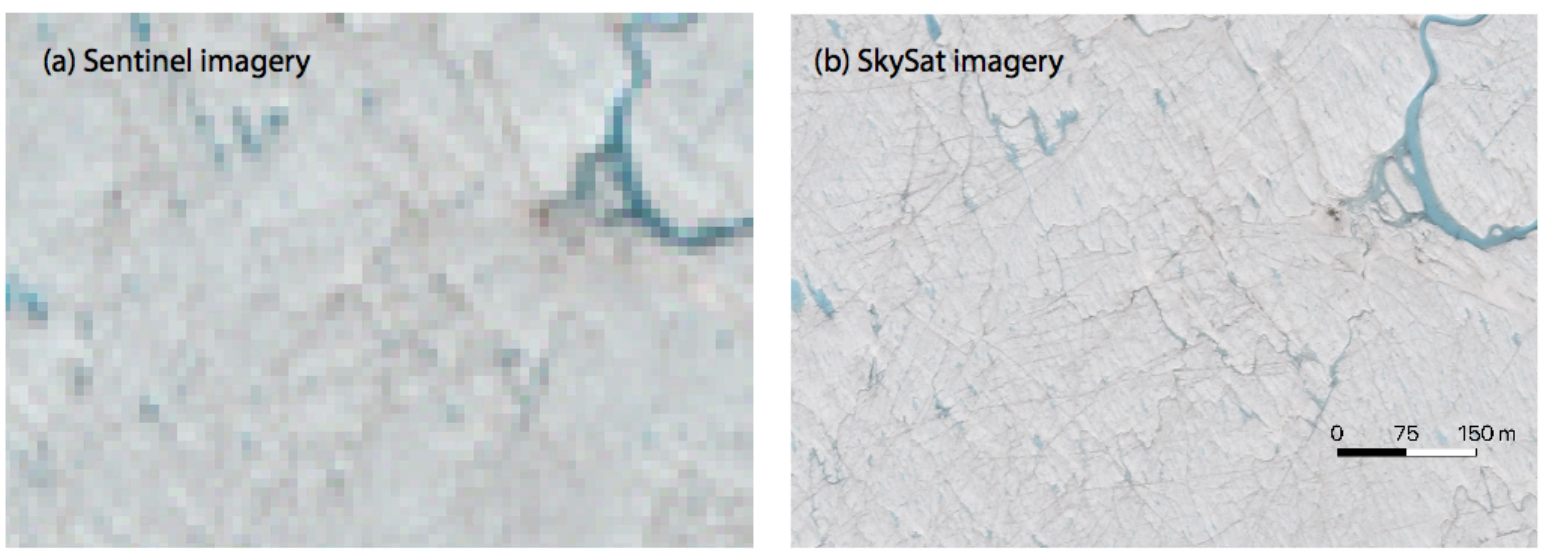

Figure S5: Higher resolution imagery capturing small-scale stream features on June $1^{\text {st }}$ in Western Greenland. (a): Sentinel-2 imagery (10m) (b): SkySat imagery 